Article

\title{
Bioclimatology, Structure, and Conservation Perspectives of Quercus pyrenaica, Acer opalus subsp. Granatensis, and Corylus avellana Deciduous Forests on Mediterranean Bioclimate in the South-Central Part of the Iberian Peninsula
}

\author{
J. C. Piñar Fuentes ${ }^{1, * \mathbb{D}}$, A. Cano-Ortiz ${ }^{1}$, C. M. Musarella ${ }^{1,2} \mathbb{D}$, R. Quinto Canas ${ }^{3,4}$, \\ C. J. Pinto Gomes ${ }^{5}$, G. Spampinato ${ }^{2}$ D, S. del Río ${ }^{6}$ and E. Cano ${ }^{1, *}$
}

1 Department of Animal and Plant Biology and Ecology, Section of Botany, University of Jaén, Campus, Universitario Las Lagunillas s/n, 23071 Jaén, Spain; anacanor@hotmail.com (A.C.-O.); carmelo.musarella@unirc.it (C.M.M.)

2 Department of AGRARIA, "Mediterranea” University of Reggio Calabria, Località Feo di Vito, 89122 Reggio Calabria, Italy; gspampinato@unirc.it

3 Faculty of Sciences and Technology, University of Algarve, Campus de Gambelas, 8005-139 Faro, Portugal; rjcanas@ualg.pt

4 Centre of Marine Sciences (CCMAR), University of Algarve, Campus de Gambelas, 8005-139 Faro, Portugal

5 Department of Landscape, Environment and Planning, Institute for Mediterranean Agrarian and Environmental Sciences (ICAAM), School of Science and Technology, University of Évora (Portugal), Rua Romão Ramalho, nº 59, 7000-671 Évora, Portugal; cpgomes@uevora.pt

6 Department of Biodiversity and Environmental Management (Botany), Mountain Livestock Institute (CSIC-ULE), Faculty of Biological and Environmental Sciences, University of León, Campus de Vegazana s/n, 24071 León, Spain; sriog@unileon.es

* Correspondence: jpinar@ujaen.es (J.C.P.F.); ecano@ujaen.es (E.C.)

Received: 13 September 2019; Accepted: 14 November 2019; Published: 18 November 2019

\begin{abstract}
The plant variability in the southern Iberian Peninsula consists of around 3500 different taxa due to its high bioclimatic, geographic, and geological diversity. The deciduous forests in the southern Iberian Peninsula are located in regions with topographies and specific bioclimatic conditions that allow for the survival of taxa that are typical of cooler and wetter bioclimatic regions and therefore represent the relict evidence of colder and more humid paleoclimatic conditions. The floristic composition of 421 samples of deciduous forests in the south-central part of the Iberian Peninsula were analyzed. The ecological importance index (IVI) was calculated, where the most important tree species were Quercus pyrenaica, Acer opalus subsp. Granatensis, and Corylus avellana. These species are uncommon in the south-central part of the Iberian Peninsula, forming forests of little extension. An analysis of the vertical distribution of the species (stratum) shows that the majority of the species of stratum 3 (hemicriptophics, camephytes, geophites, and nanophanerophytes) are characteristic of deciduous forests, and their presence is positively correlated with high values of bioclimatic variables related to humidity and presence of water in the soil (nemoral environments), while they are negatively correlated with high values of bioclimatic variables related to high temperatures, evapotranspiration, and aridity. This work demonstrates that several characteristic deciduous forest taxa are more vulnerable to disappearance due to the loss of their nemoral conditions caused by gaps in the tree or shrub canopy. These gaps lead to an increase in evapotranspiration, excess insolation, and a consequent loss of water and humidity in the microclimatic conditions.
\end{abstract}

Keywords: global warming; bioclimate; deciduous forests; southern Iberian Peninsula; sustainable management; multivariable analyses 


\section{Introduction}

It has been proven that territories behave differently in response to the general climate, substrate type, and the topography of the terrain [1]. Bioclimatology is defined as the geobotanical science that studies the reciprocity between climate and the distribution of living beings and their communities on Earth and evidently has much to offer in these cases. This discipline, also known as phytoclimatology, emerged as a result of the connection between numerical climate values (temperature and precipitation) and the distribution areas of vegetation species, plant communities, ecosystems, and biomes [2]. The indices of Rivas Martínez [3] have established a close correlation between bioclimatic data and plant communities, enabling each territory to be characterised in these terms [4].

This bioclimatic approach can be applied to a range of crops under a Mediterranean macro-bioclimate. Different woodlands can be defined taking into account these bioclimatic aspects according to their climate dependence. Woodlands on rocky substrates and shallow soils with low water-retention capacity occur on soils or in biotopes that are especially dry or xerophytic (edaphoxerophilous woodlands). Climatophilous woodlands are found in mature soils in the corresponding ombroclimate that receive only rainwater. Temporihygrophilous, mesophytic, and mesohygrophytic series have an exceptional water intake for topographic reasons. Finally, edaphohygrophilous woodlands occupy especially humid soils and are found in riverbeds, marshes, peat bogs, and similar environments and have a higher humidity corresponding to the ombroclimate [5].

The combination of drought and shade evidently leads to greater photoinhibition and water deficit in the shade than in the sun [6], which seriously affects plant recruitment and the regeneration of the natural forest. This is partly due to dimming caused by the rise in global temperatures. The reduction in the amount of light as a result of the abandonment of Mediterranean woody ecosystems, together with the anticipated global dimming [7], is likely to cause a decrease in the spatial heterogeneity of light and the gradual disappearance of shrub communities characteristically found where there is an abundance of light, which in turn could lead to impoverished forests and gaps with only a layer of herbaceous understorey [8].

Several authors have related a range of abiotic and biotic variables with germination rates, seedling establishment, and the overall development of different species in the plant communities. Interannual climate variation can alter the quality of microhabitats for the establishment of seedlings in heterogeneous Mediterranean forests [9]. The emergence and survival of seedlings is facilitated by several mechanisms, including a reduction in radiation and soil desiccation $[10,11]$; litterfall production, which decreases water loss from evaporation and favours the survival of the seedlings and juveniles in the plant species [12,13]; reduction in soil compaction [14]; and an increase in soil macroporosity and water storage [15]. These are all [16] determining factors in the recruitment, establishment, and regeneration of forests.

Another aspect to take into account for an understanding of forest dynamics is the role played by nurse plants, generally hemicryptophytes, chamaephytes, and micro- and mesophanerophytes, which act as forest edges and are distributed on a gradient from pre-forest edges through to non-forest biotopes (normally grasslands). This dense, mid-height perennial non-nitrophilous vegetation is found in contact with well-conserved forests or pre-forest sites. The environment in these communities is a transition between access to light, the refugium effect of the ecoclimate and the soil processes on the forest margins, which favours the more mature and climactic stages [17].

Studies on the various deciduous species present in the heart of the Mediterranean bioclimatic domain highlight a problem of recruitment and regeneration $[9,18]$ and confirm that interannual climate variation can alter the quality of microhabitats for the germination and establishment of seedlings in Mediterranean forests of Acer opalus subsp. granatense (Boiss.) Font Quer and Rothm., which is highly dependent on the microhabitat, and is much lower in open spaces than under established tree or shrub 
vegetation [19]. A decline in mesophytic conditions due to gaps in the vegetation increases the levels of direct evapotranspiration and leads to the establishment of plant communities composed of more xerophytic taxa [20]. It is vitally important for the regeneration and dynamics of the Corylus avellana $\mathrm{L}$. formations in the southern Iberian Peninsula to conserve the subserial shrubs formed by dense closed thorn bushes that shelter many mature forest species [21].

Other studies on deciduous tree species such as Quercus pyrenaica Willd. and Quercus canariensis Willd. have produced similar results. Q. pyrenaica requires colder environments with higher year-round precipitation, meaning that forests dominated by Q. pyrenaica are often in contact with other marcescent forests where there is an absence of optimal conditions for the development of deciduous forests [22]. These climate conditions may be an essential factor in allowing germination practically all year round [23]. Q. canariensis has a lower rate of germination compared to other deciduous species in the Quercus genus, although the establishment of these seedlings largely depends on the availability of water in the driest months [24].

The typical vegetation of the Mediterranean bioclimate has adapted to these characteristic climate fluctuations. Quercus rotundifolia Lam. occurs in a Mediterranean environment with summer drought and so has logically developed strategies that allow it to complete its germination before the start of the dry season [23]. Another typical species of this bioclimate, Quercus suber L., has been shown by other authors [24] to have its own mechanisms for withstanding water stress, whereby an increase in water availability in summer does not lead to greater photosynthetic activity.

The aims of this work is to relate the structure and floristic composition of the deciduous forests of Quercus pyrenaica, Acer opalus subsp. Granatensis, and Corylus avellana with the different bioclimatic variables involved in these deciduous formations that appear in the middle of the Mediterranean bioclimate and to relate the different taxa in the deciduous forests in the southern Iberian Peninsula with the bioclimatic variables that define their presence or absence. This is intended to answer questions such as which species would be more vulnerable to disappear due to global warming. The vertical structure of these forests behaves similarly. Another issue is to observe what bioclimatic variables are involved in the floristic composition of the different forest stratum.

\section{Materials and Methods}

\subsection{Study Subjects}

This work studies the forest formations dominated by Acer opalus subsp. granatense, Corylus avellana, and Quercus pyrenaica in the southern half of the Iberian Peninsula (Figure 1a). The study area comprises the more humid and relatively cool areas in the southern Iberian Peninsula that are completely contained within the Mediterranean bioclimatic region.

From a bioclimatic point of view, these deciduous formations are distributed throughout the thermo-, meso-, and supramediterranean bioclimatic belts, with an ombrotype ranging from the lower sub-humid to the upper humid [5]. The continentality index for these forest formations varies from the attenuated continental-subcontinental for the northernmost and easternmost formations to oceanic semi-hyperoceanic for formations with a more southerly distribution and a clear influence of the Atlantic Ocean.

The biogeography for these plant communities comprises the Mediterranean region are included in the Holarctic biogeographical realm. Within this region, the formations in the study are distributed in the Luso-Extremaduran, Baetic, Gaditano-Onubo-Algarvian, and Iberian Central Mediterranean provinces (Figure 1b).

These deciduous formations are established on a very diverse range of geological materials, including basic materials such as limestone, limestone-dolomite for formations dominated by Acer opalus subsp. granatense and Corylus avellana, to siliceous materials such as quartzite, sandstone, slate, schists and mica schists, where most of the Quercus pyrenaica formations occur. 


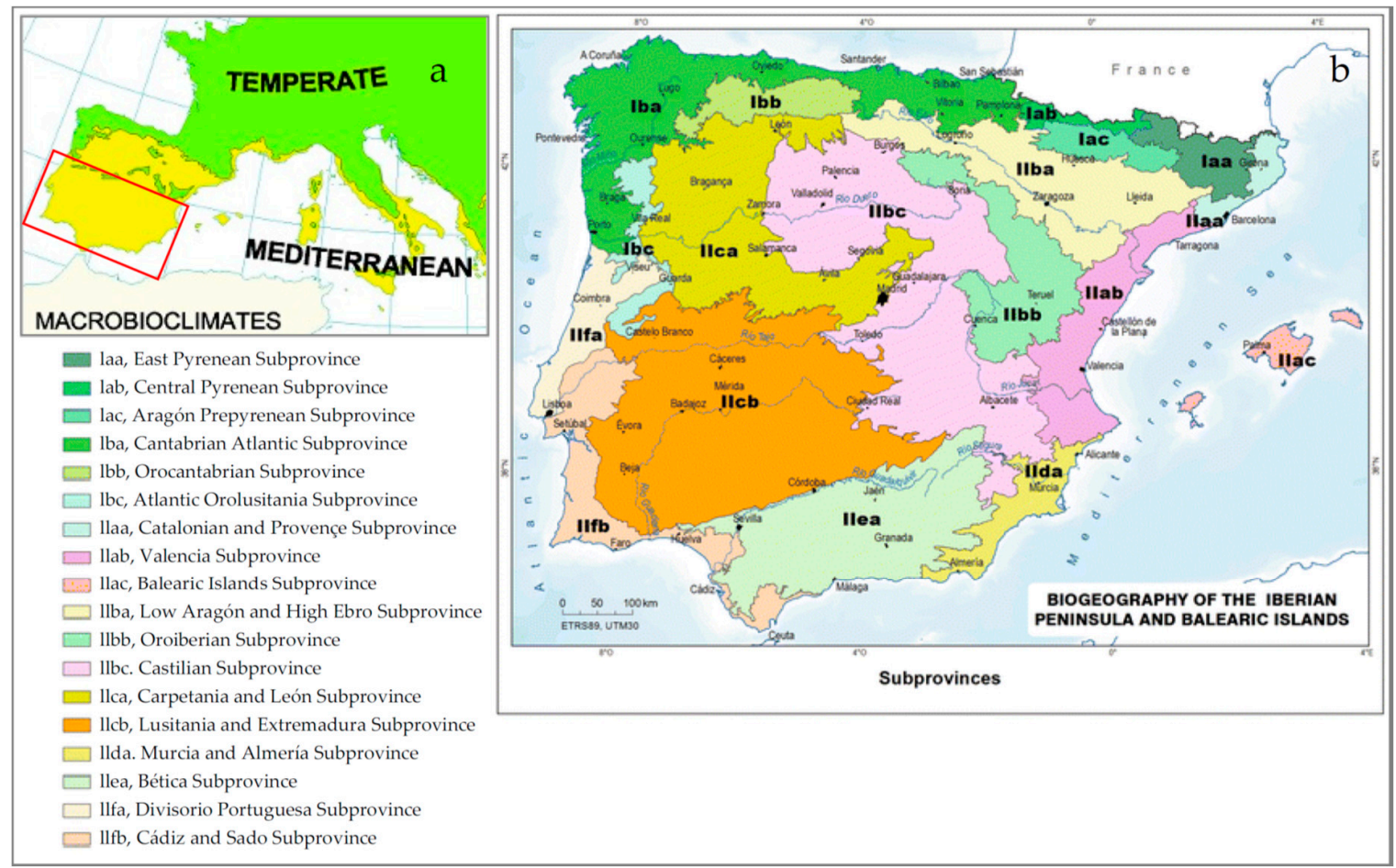

Figure 1. Location of the study area (red rectangle): (a) macrobioclimate map of Europe. All the relevés are located in the Mediterranean macrobioclimate (adapted from [25]; (b) biogeographic map of the Iberian Peninsula. The sampling points belong to the subprovinces: llea, llcb, llfb, and llca. Adapted from reference [26].

\subsection{Origin and Structure of the Vegetation Data}

We use a total of 421 vegetation relevés distributed as follows: 22 of our own unpublished relevés and relevés from the bibliography (399 relevés) (Table 1), with the greatest presence and abundance of the abovementioned deciduous species, to analyse the vulnerability of these plant communities. These relevés were compiled following the abundance/dominance phytosociological method [27]. The phytosociological abundance indices with a qualitative or semi-quantitative character are transformed and standardised to average cover percentages as shown in Table 2. Subsequently, and as a selection criterion for the different taxa, the importance value index (IVI) was calculated according to the following formula:

$$
I V I=D R+F R+D o R
$$

where $D R$ is the relative density.

$$
D R_{i}=\left(\frac{c_{i}}{C t}\right) * 100
$$

where $c$ is the cover of species $i$ and $C t$ is the sum of the total cover of all the species.

$F R$ is the relative frequency of each species.

$$
F R_{i}=\frac{F A i}{\sum F A} * 100
$$

where $F A$ is the absolute frequency of each species.

$\mathrm{DoR}$ is the relative dominance, closely linked to its life form and growth habit.

$$
D o R_{i}=\frac{s_{i}}{\sum_{1}^{i} s_{i}}
$$

where $s_{i}$ is the projected area at ground level of each species. 
Table 1. Origin of the vegetation samples used in this work.

\begin{tabular}{|c|c|c|}
\hline Plant Community & Bibliography & Number of Relevés \\
\hline \multirow{6}{*}{ AdQp } & El Aallali et al. [33] & 12 \\
\hline & Losa et al. [34] & 1 \\
\hline & Martínez Parras \& Molero [28] & 15 \\
\hline & Nieto \& Cabezudo [35] & 3 \\
\hline & Pérez Latorre et al. [36] & 5 \\
\hline & Junta de Andalucía. [37] & 25 \\
\hline \multirow{11}{*}{$\mathrm{AuQp}$} & Rivas Goday [38] & 14 \\
\hline & Cano E. \& Valle F. [39] & 6 \\
\hline & Melendo Luque [40] & 3 \\
\hline & Vicente Orellana \& Galán De Mera [41] & 11 \\
\hline & Own unpublished relevés & 16 \\
\hline & Amor, A.; [42] & 9 \\
\hline & Belmonte M.D.; [43] & 12 \\
\hline & Monteiro-Henriques, T.; [44] & 6 \\
\hline & Pereira [45] & 10 \\
\hline & Sánchez-Pascual, N. [46] & 8 \\
\hline & Junta de Andalucía. [37] & 48 \\
\hline \multirow{3}{*}{ BhQp } & Valle et al. [30] & 8 \\
\hline & López Vélez, G. [47] & 7 \\
\hline & Herranz et al. [48] & 3 \\
\hline \multirow{3}{*}{ LbQp } & Galán de Mera, A. [49] & 5 \\
\hline & Pérez Latorre et al. [50] & 4 \\
\hline & Asensi, A.; Díaz Garreta, B.; [51] & 1 \\
\hline \multirow{5}{*}{ StQp } & Rivas Goday, S. [52] & 13 \\
\hline & Cantó, Paloma. [53] & 20 \\
\hline & Own unpublished relevés & 6 \\
\hline & Sánchez-Pascual, N. [46] & 3 \\
\hline & Junta de Andalucía. [37] & 1 \\
\hline \multirow{16}{*}{ DlAg } & Cabezudo et al. [54] & 1 \\
\hline & Giménez Luque, Esther; [55] & 5 \\
\hline & Gómez Mercado et Valle [56] & 5 \\
\hline & Gómez-Mercado, F.; [57] & 22 \\
\hline & Gómez Mercado et al. [58] & 4 \\
\hline & Herranz et al. [49] & 14 \\
\hline & López Vélez, G.; [48] & 10 \\
\hline & Madrona, M.T.; [59] & 2 \\
\hline & Pavón \& Pérez Latorre [60] & 5 \\
\hline & Pérez-Latorre et al. [61] & 1 \\
\hline & Inocencio Pretel [62] & 1 \\
\hline & Olmedo, J.A.; [63] & 3 \\
\hline & Gómez Navarro, José; [64] & 3 \\
\hline & Molina Cantos et al. [65] & 3 \\
\hline & Pavón Núñez et al. [66] & 1 \\
\hline & Junta de Andalucía. [37] & 32 \\
\hline \multirow{6}{*}{$\mathrm{GuCa}$} & Gómez-Mercado, F.; [58] & 3 \\
\hline & López Vélez, G.; [47] & 8 \\
\hline & Pavón \& Pérez Latorre [60] & 5 \\
\hline & Ríos Ruiz et al. [67] & 7 \\
\hline & Valle et al. [21] & 8 \\
\hline & Junta de Andalucía. [37] & 3 \\
\hline
\end{tabular}

Adenocarpo decorticantis-Quercetum pyrenaicae (AdQp); Arbuto unedonis-Quercetum pyrenaicae (AuQp); Berberido australis-Quercetum pyrenaicae (BhQp); Luzulo baeticae-Quercetum pyrenaicae (LbQp); Sorbo torminalis-Quercetum pyrenaicae (StQp); Daphno latifoliae-Aceretum granatensis (DlAg) and Geo urbani-Coryletum avellanae (GuCa). 
Table 2. Conversion of the values of the phytosociological indices and their correspondence with the average percentage cover values.

\begin{tabular}{cc}
\hline Phytosociological Index & \% of Average Cover \\
\hline 5 & 85 \\
4 & 65 \\
3 & 45 \\
2 & 25 \\
1 & 12 \\
+ & 6 \\
r & 3
\end{tabular}

The non-numerical values (" + " and " $r$ ") have been transformed and estimated by interpolation from the numerical indices.

The phytosociological nomenclature for these plant communities is Adenocarpo decorticantis-Quercetum pyrenaicae Martínez-Parras and Molero 1982 [28] (AdQp); Arbuto unedonis-Quercetum pyrenaicae (Rivas Goday in Rivas Goday, Esteve, Galiano, Rigual and Rivas-Martínez 1960) Rivas-Martínez 1987 [29] (AuQp); Berberido australis-Quercetum pyrenaicae F. Valle, Gómez-Mercado and Mota 1988 [30] (BhQp); Luzulo baeticae-Quercetum pyrenaicae Rivas-Martínez 2002 [31] (LbQp); Sorbo torminalis-Quercetum pyrenaicae Rivas Goday ex Rivas-Martínez 1987 [29] (StQp); Daphno latifoliae-Aceretum granatensis Rivas-Martínez 1965 [32] (DlAg) and Geo urbani-Coryletum avellanae F. Valle, Mota and Gómez-Mercado 1986 [21] (GuCa).

\subsection{Climatic, Geographic, and Topographic Data}

Subsequently, and based on the geographic and topographic information contained in these relevés, each sampling was geo-referenced and implemented in a geographic information system (GIS) using the corresponding maps. They were then matched with the place names and the rest of the data from the samplings in the bibliography in order to obtain the different climatic and bioclimatic variables for each sampling.

The figures for altitude, orientation and slope were obtained by plotting the previously geo-referenced points on a digital terrain map (DTM) with a pixel resolution of 30x30 metres for the points in Portugal and Morocco and on a digital terrain model with a resolution of 10x10 metres for the points in Spain. The numerical values for altitude, orientation, and slope were obtained from the DTM for each sampling point.

The climate and bioclimatic variables were defined following the criterion of Rivas-Martínez [5]. Climate variables can be divided into

a. Climate variables. The following climate variables have been considered for the present study:

T: Mean annual temperature in degrees centigrade

M: Mean temperature of the maximums

$\mathrm{m}$ : Mean temperature of the minimums

Tmax: Mean temperature of the warmest month of the year

Tmin: Mean temperature of the coldest month of the year

Tcmax: Mean maximum temperature of the month with the greatest temperature difference in the year Tcmin: Mean minimum temperature of the month with the greatest temperature difference in the year Tn: Negative annual temperature (sum of the months with a mean temperature below $0^{\circ} \mathrm{C}$ in tenths of degrees centigrade)

Tp: Positive annual temperature (sum of the months with a mean temperature above $0{ }^{\circ} \mathrm{C}$ in tenths of degrees centigrade)

Tpd: Positive temperature of the driest quarter of the year, in tenths of degrees centigrade $\mathrm{Tpd}_{1}$ : Positive temperature of the driest month of the year, in tenths of degrees centigrade 
$\mathrm{Tpd}_{2}$ : Positive temperature of the driest two months of the year, in tenths of degrees centigrade Pp: Positive annual precipitation (of the months in Ti above $0{ }^{\circ} \mathrm{C}$ )

PE: Thornthwaite's potential annual evapotranspiration index PEs: Potential evapotranspiration index of the summer quarter $\mathrm{PE}_{1-12}$ : Potential monthly evapotranspiration index $1=$ January, $2=$ February, $\ldots 12=$ December

b. Bioclimatic variables used:

Annual aridity index (Iar);

$$
\operatorname{Iar}=\frac{P E}{P}
$$

Diurnality index $(I d)$ : the difference between the mean maximum temperature (Tc max) and the mean minimum temperature (Tc $\mathrm{min}$ ) of the months with the greatest temperature differences in the year; that is, the month with the greatest daily swing or interval between maximum and minimum temperatures.

$$
I d=\text { Tcmax }- \text { Tcmin }
$$

Humidity index: the percentage of precipitation above or below the annual precipitation $(P)$ compared to the annual evapotranspiration $(P E)$

$$
I H=100 * \frac{(P-P E)}{P E}
$$

Mediterraneity index (Im): the coefficient between the value for Thornthwaite's mean summer evapotranspiration $(P E s)$, and precipitation in $\mathrm{mm}$ in the same period $(P s)$.

$$
I m=\frac{P E S}{P S}
$$

Mediterraneity index for the month of July $\left(I m_{1}\right)$ and for July and August $\left(I m_{2}\right)$;

$$
I m_{1}=\frac{P E s_{7}}{P s_{7}} ; I m_{2}=\frac{P E s_{7-8}}{P s_{7-8}}
$$

Annual evapotranspiration index (Ioe): the coefficient between mean precipitation $(P)$ and potential annual evapotranspiration ( $P E$, Thornthwaite).

$$
\text { Ioe }=\frac{P}{P E}
$$

Ombrothermic index of the warmest month of the summer quarter $\left(I o s_{1}\right)$ : ombrothermic index of the warmest two months of the summer quarter $\left(\operatorname{Ios}_{2}\right)$, ombrothermic index of the summer quarter (Ios3), and ombrothermic index of the summer quarter and the previous month $\left(\operatorname{Ios}_{4}\right)$.

$$
\operatorname{Ios}_{1}=10 * \frac{P_{\text {warmest month }}}{T_{m} \text { warmest month }} ; \operatorname{Ios}_{2}=10 * \frac{P_{\text {warmest two months }}}{T_{\text {warmest two months }}} ; \operatorname{Ios}_{3}=10 * \frac{P_{6-8}}{T_{6-8}} ; \operatorname{Ios}_{4}=10 * \frac{P_{5-8}}{T_{5-8}}
$$

Monthly ombrothermic indices $\left(\operatorname{Iom}_{1-12}\right)$, with $\mathrm{i}=1=$ January $2=$ February, $\ldots, 12=$ December.

$$
\operatorname{Iom}_{i}=\frac{P_{i}}{T_{i}}
$$


Annual ombrothermic index (Io): the coefiicient between the total mean precipitation in $\mathrm{mm}$ in the months with a mean temperature above zero degrees centigrade $(P p)$ and the sum of the mean monthly temperatures above zero degrees in tenths of a degree $(T p)$.

$$
I o=\frac{P p}{T p}
$$

Simple continentality index (Ic): the continentality index expresses in degrees centigrade the difference or oscillation between the mean temperature of the warmest month (Tmax) and the mean temperature of the coldest month of the year (Tmin).

$$
\text { Ic }=\text { Tmax }- \text { Tmin } .
$$

Thermicity index (It): this index describes the intensity of cold, which is a limiting factor for many plants and plant communities.

$$
I t=(T+m+M) 10
$$

To weight the excess of cold or thermicity in the most continental or oceanic locations in areas located north or south of parallel $23^{\circ}$, a compensation factor C (Table 3 ) is added to the It value based on the continentality values in the area, Itc $=$ It $+C$.

Table 3. Calculation of the compensation value of C, for the calculation of ITC, based on the Ic value.

\begin{tabular}{cc}
\hline Ic Value & C Value \\
\hline$<=8$ & $\mathrm{C}=(\mathrm{Ic}-8) \times 10$ \\
$8<$ Ic $<=18$ & $\mathrm{C}=0$ \\
$18<$ Ic $<=21$ & $\mathrm{C}=(\mathrm{Ic}-18) \times 5$ \\
$21<$ Ic $<=28$ & $\mathrm{C}=(\mathrm{Ic}-21) \times 15$ \\
$28<$ Ic $<=46$ & $\mathrm{C}=(\mathrm{Ic}-28) \times 25$ \\
$46<$ Ic $<=65$ & $\mathrm{C}=(\mathrm{Ic}-46) \times 60$ \\
\hline
\end{tabular}

The interpolation of the climate data for precipitation and temperature for each sampling point was obtained from the data from 3192 weather stations distributed throughout the southern half of the Iberian Peninsula and the north of Africa from the National Meteorological Agency (AEMET), the Climate Information System of the Andalusian Regional Government, the Integral Irrigation Advisory Service of Castile-La Mancha (SIAR), the Murcia Agricultural Information System (SIAM), and the Extremadura Irrigation Advisory Network (REDAREX). The precipitation and temperature values were obtained for each sampling site using geostatistical interpolation methods based on kriging and cokriging. The bioclimatic variables described above were subsequently calculated from the interpolated values.

Explanatory factor analysis is the name used to describe a series of multivariate statistical methods of interdependence for the purpose of identifying a structure of factors underlying a broad set of data in order to establish which bioclimatic variables (in this case) have the greatest relevance. A factor analysis was carried out with these data, and the main components were extracted; that is, the variables that best explain the variability along the first two axes (see Table 4).

Spearman's correlation method was chosen because it is robust against the independence of the distribution of each of the variables involved. 
Table 4. Assignment of the variables with the highest factor coordinates to each of the first two axes.

\begin{tabular}{cccccccccccccccccc}
\hline Axis & Tmax & Tmin & Tp & PEs & PE & Iar & Id & IH & Ioe & Ios $_{\mathbf{1}}$ & Ios $_{\mathbf{2}}$ & Ios $_{\mathbf{3}}$ & Ios $_{\mathbf{4}}$ & Ic & Io & Itc & \\
\hline Axis1 & 0.60 & 0.55 & 0.63 & 0.56 & 0.65 & 0.44 & 0.21 & -0.44 & -0.44 & -0.36 & -0.30 & -0.22 & -0.33 & 0.01 & -0.50 & 0.63 & 0.53 \\
Axis2 & -0.36 & -0.57 & -0.46 & -0.43 & -0.43 & -0.37 & 0.15 & 0.37 & 0.37 & 0.54 & 0.56 & 0.58 & 0.55 & 0.29 & 0.42 & -0.45 \\
\hline
\end{tabular}

The climate or bioclimatic variables $\mathrm{Im}_{1} \mathrm{Im}_{1}, \mathrm{Im}_{2}, \mathrm{P}, \mathrm{Pp}, \mathrm{T}, \mathrm{M}, \mathrm{m}$, Tcmax, Tcmin, $\mathrm{Iom}_{1-12}$, $\mathrm{Tpd}, \mathrm{Tpd}_{1}$, and $\mathrm{Tpd}_{2}$ were discarded as they presented collinearity and had their maximum coordinates on axes other than axes 1 and 2 . 


\subsection{Analysis of the Subjects (Relevés from Deciduous Forests)}

Due to the high number of taxa in all the relevés (546 taxa), they were filtered taking into account their adscription to one of the following phytosociological classes: Querco roboris-Fagetea sylvaticae Br.-Bl. and Vlieger in Vlieger 1937 [68]; Quercetea ilicis Br.-Bl. Ex A. and O. Bolós 1950 [69]; and Salici purpureae-Populetea nigrae Rivas-Martínez et al., 2001 [70]. These phytosociological classes include the characteristic vegetation of the deciduous and sclerophyllous forests of the southern Iberian Peninsula. The different forest strata were defined following a criterion based on their life form, as follows: macroand mesophanerophytes were assigned to stratum 1 (E1); micro- and nanophanerophytes and vines were assigned to stratum 2 (E2); chamaephytes, geophytes, and hemicryptophytes to stratum 3 (E3); and therophytes to stratum 4 (E4). The different taxa were assigned to each phytosociological class following the published list of Rivas-Martínez [71] and the IVI value. The result of this adscription can be seen in Tables 4 and A1. The climate variables selected had a maximum square cosine on one of the first two axes with the highest variability. A canonical correspondence analysis (CCA) was carried out to relate the climate variables with the different species. The possible correlation between the abundance of characteristic taxa of Quercetea ilicis and the different bioclimatic variables was determined by means of an analysis of variance (ANOVA) after a Shapiro-Wilk normality test to confirm that the data meets the assumptions to ensure the reliability of the ANOVA test.

\section{Results}

\subsection{Correlation between Characteristic Taxa, Stratum, and Bioclimatic Variables}

\subsubsection{Spearman's Correlation Analysis}

There are two large groups of taxa, as can be seen in Table 3. The first group comprises taxa that are negatively correlated with temperature-related bioclimatic variables such as mean maximum (Tmax) or mean minimum temperature (Tmin). These variables show a significant negative correlation with the characteristic taxa of Querco roboris-Fagetea sylvaticae, which include these deciduous formations. On the other hand, as can be seen in Figure 2, the ratio of species that are on different stratum that structure this deciduous forests, depending on their correlation with the bioclimatic indices in a negative or positive way, changes quite a lot depending on the stratum E1, E2, E3, or E4 in which they are part of the forest. These species have their optimum in colder regions with mild summers and therefore with lower summer evapotranspiration (PEs) and annual evapotranspiration (PE) values [71]. This can be seen in the correlation coefficients between the abovementioned species and the bioclimatic variables PEs and PE in places or microclimates where the abundance or presence of these mesophytic species (which, for the most part, are part of E3), declines when the values of PEs and PE increase, either due to high temperatures [72] or to gaps in the vegetation [20,21].

Table 5 also shows that these taxa have a significant positive correlation with the humidity index (IH), the annual ombrothermic index (Io) and the summer ombrothermic indices: the ombrothermic index of the warmest month in the summer quarter $\left(\operatorname{Ios}_{1}\right)$, the warmest two months in the summer quarter $\left(\operatorname{Ios}_{2}\right)$, the summer quarter $\left(\operatorname{Ios}_{3}\right)$, and the summer quarter plus the previous month (May in the northern hemisphere) $\left(\operatorname{Ios}_{4}\right)$ [73]. 


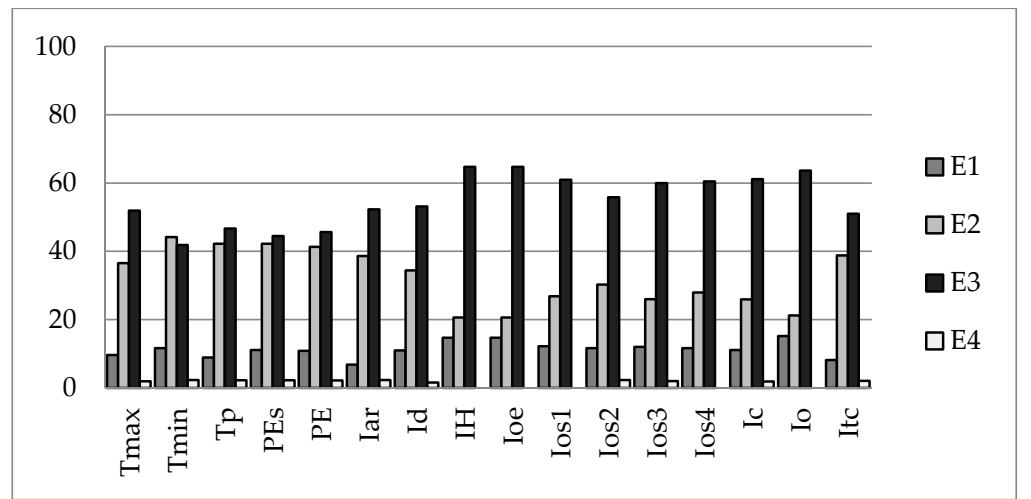

(a)

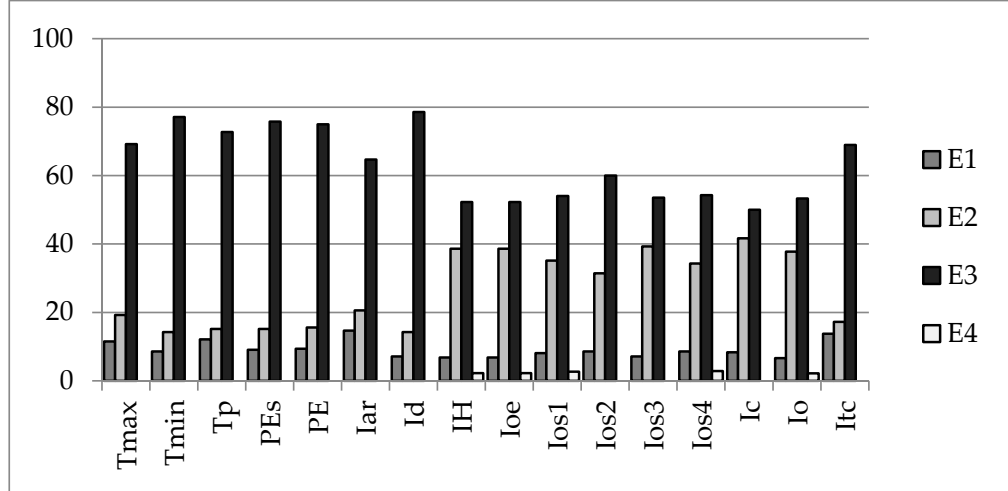

(b)

Figure 2. Distribution by strata (E1-E4) of the different characteristic taxa that are part of the deciduous forests, depending on their correlation with the different bioclimatic variables involved. (a) Distribution of the species by strata positively correlated with the bioclimatic variables (the highest proportion of E3 taxa stands out in the variables related to moisture and soil water compensation). (b) Distribution by species negatively correlated with the bioclimatic variables (it is worth highlighting the greater proportion of E3 taxa correlated negatively to the bioclimatic parameters related to temperature, aridity and evapotranspiration).

The annual ombro-evaporation index (Ioe) is of great importance for taxa growing in nemoral and more humid environments especially for species that grow in stratum E2 and E3 and therefore with lower water loss due to evapotranspiration [73-75]. Taxa such as Primula acaulis, Daphne laureola subsp. latifolia, Ilex aquifolium, Corylus avellana L. Paeonia officinalis subsp. microcarpa, Helleborus foetidus, Quercus faginea subsp. faginea, Hedera helix subsp. helix, Acer opalus subsp. granatense, Luzula forsteri subsp. forsteri, Quercus faginea subsp. alpestris, Viola sylvestris subsp. sylvestris, Ulmus glabra, Epipactis microphylla, Euphorbia hyberna, Carex sylvatica, and Solidago virgaurea are widely abundant or have preferences for biotopes with microclimatic sites with a higher Ioe.

There is a significant positive correlation between the bioclimatic indices related to annual potential evapotranspiration (PE), summer potential evapotranspiration (PEs), and the aridity index (Iar) [73] and taxa such as Tamus communis, Teucrium scorodonia subsp. scorodonia, Quercus faginea subsp. faginea, Luzula forsteri subsp. forsteri, Allium massaessylum, Acer monspessulanum subsp. monspessulanum, Holcus mollis subsp. mollis, Epipactis helleborine subsp. Helleborine, and Arenaria montana subsp. montana. 
Table 5. Spearman's correlation coefficients between bioclimatic indices and indices for the abundance of taxa.

\begin{tabular}{|c|c|c|c|c|c|c|c|c|c|c|c|c|c|c|c|c|c|c|c|}
\hline Species & $\begin{array}{c}\text { Phytosociological } \\
\text { Class }\end{array}$ & Stratum & IVI & Tmax & Tmin & $T p$ & PEs & PE & Iar & Id & IH & Ioe & Ios1 & Ios2 & Ios3 & Ios4 & Ic & Io & Itc \\
\hline Quercus pyrenaica Willd. & Q-F & E1 & 12.63 & 0.25 & 0.29 & 0.31 & 0.22 & 0.31 & 0.30 & -0.10 & -0.30 & -0.30 & -0.28 & -0.30 & -0.29 & -0.34 & -0.07 & -0.32 & 0.32 \\
\hline $\begin{array}{l}\text { Acer opalus subsp. granatense } \\
\text { (Boiss.) Font Ouer and Rothm. }\end{array}$ & Q-F & E2 & 6.31 & -0.38 & -0.42 & -0.45 & -0.37 & -0.45 & -0.13 & -0.09 & 0.13 & 0.13 & 0.27 & 0.24 & 0.19 & 0.20 & 0.11 & 0.19 & -0.45 \\
\hline Corylus avellana $\mathrm{L}$. & Q-F & E2 & 5.04 & -0.10 & -0.12 & -0.07 & -0.03 & -0.06 & -0.28 & 0.28 & 0.28 & 0.28 & 0.34 & 0.34 & 0.36 & 0.35 & 0.03 & 0.27 & -0.11 \\
\hline $\begin{array}{l}\text { Quercus faginea subsp. faginea } \\
\text { Lam. }\end{array}$ & Q-F & E1 & 4.78 & 0.08 & 0.04 & 0.07 & 0.12 & 0.08 & -0.18 & 0.33 & 0.18 & 0.18 & 0.22 & 0.30 & 0.24 & 0.22 & 0.07 & 0.16 & 0.03 \\
\hline $\begin{array}{l}\text { Brachypodium sylvaticum (Huds.) } \\
\text { P. Beauv. }\end{array}$ & S-P & E3 & 4.44 & 0.14 & 0.09 & 0.16 & 0.13 & 0.17 & -0.09 & 0.26 & 0.09 & 0.09 & 0.09 & 0.10 & 0.18 & 0.14 & 0.07 & 0.08 & 0.15 \\
\hline Rubia peregrina subsp. peregrina $\mathrm{L}$. & Q-I & E2 & 4.40 & 0.18 & 0.25 & 0.25 & 0.21 & 0.26 & -0.04 & 0.24 & 0.04 & 0.04 & 0.03 & 0.04 & 0.11 & 0.05 & -0.03 & 0.01 & 0.24 \\
\hline $\begin{array}{l}\text { Daphne laureola subsp. latifolia } \\
\text { (Coss.) Rivas Mart. }\end{array}$ & Q-F & E3 & 4.24 & -0.25 & -0.33 & -0.29 & -0.23 & -0.29 & -0.42 & 0.19 & 0.42 & 0.42 & 0.38 & 0.41 & 0.37 & 0.40 & 0.22 & 0.43 & -0.32 \\
\hline $\begin{array}{l}\text { Helleborus foetidus L. } \\
\text { Arbutus unedo L. }\end{array}$ & $\begin{array}{l}\text { Q-I } \\
\text { Q-F }\end{array}$ & $\begin{array}{l}\text { E3 } \\
\text { E2 }\end{array}$ & $\begin{array}{l}4.18 \\
4.18\end{array}$ & $\begin{array}{c}-0.29 \\
0.46\end{array}$ & $\begin{array}{c}-0.30 \\
0.44\end{array}$ & $\begin{array}{c}-0.32 \\
0.49\end{array}$ & $\begin{array}{c}-0.26 \\
0.50\end{array}$ & $\begin{array}{c}-0.32 \\
0.51\end{array}$ & $\begin{array}{c}-0.34 \\
0.16\end{array}$ & $\begin{array}{l}0.05 \\
0.22\end{array}$ & $\begin{array}{c}0.34 \\
-0.16\end{array}$ & $\begin{array}{c}0.34 \\
-0.16\end{array}$ & $\begin{array}{c}0.24 \\
-0.17\end{array}$ & $\begin{array}{c}0.23 \\
-0.17\end{array}$ & $\begin{array}{c}0.22 \\
-0.07\end{array}$ & $\begin{array}{c}0.27 \\
-0.05\end{array}$ & $\begin{array}{l}0.06 \\
0.05\end{array}$ & $\begin{array}{c}0.35 \\
-0.21\end{array}$ & $\begin{array}{c}-0.32 \\
0.46\end{array}$ \\
\hline Daphne gnidium $\mathrm{L}$. & Q-I & E3 & 3.62 & 0.34 & 0.42 & 0.43 & 0.36 & 0.42 & 0.13 & 0.13 & -0.13 & -0.13 & -0.23 & -0.18 & -0.10 & -0.13 & -0.07 & -0.17 & 0.42 \\
\hline $\begin{array}{l}\text { Quercus faginea subsp. alpestris } \\
\text { (Boiss.) Maire }\end{array}$ & Q-F & E1 & 3.60 & -0.13 & -0.14 & -0.11 & -0.10 & -0.11 & -0.24 & 0.07 & 0.24 & 0.24 & 0.20 & 0.22 & 0.20 & 0.23 & 0.04 & 0.24 & -0.13 \\
\hline Paeonia broteri Boiss. \& Reut. & Q-F & E3 & 3.52 & 0.23 & 0.01 & 0.13 & 0.27 & 0.17 & 0.18 & 0.21 & -0.18 & -0.18 & 0.10 & 0.17 & 0.18 & 0.07 & 0.31 & -0.19 & 0.08 \\
\hline Tamus communis $\mathrm{L}$. & 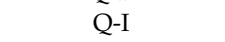 & E & 3.51 & 0.2 & 0.3 & 0.3 & 0.3 & 0.3 & 0.0 & 0.22 & -0.06 & -0.06 & -0.12 & -0.06 & 0.0 & 0.00 & 0.00 & -0.11 & 0.34 \\
\hline Hedera helix & $\mathrm{Q}-\mathrm{F}$ & E2 & 3.2 & -0.09 & -0.11 & -0.08 & -0.03 & -0.06 & -0.28 & 0.24 & 0.28 & 0.28 & 0.29 & 0.31 & 0.31 & 0.31 & 0.06 & 0.27 & -0.10 \\
\hline Quercus sube & Q-I & E1 & 3.2 & 0.35 & 0.49 & 0.50 & 0.37 & 0.48 & 0.11 & 0.13 & -0.11 & -0.11 & -0.23 & -0.31 & -0.20 & -0.18 & -0.18 & -0.17 & 0.49 \\
\hline Myrtus communis L. & Q-I & E2 & 3.12 & 0.07 & 0.14 & 0.14 & 0.08 & 0.14 & 0.1 & 0.12 & -0.10 & -0.10 & -0.09 & -0.15 & -0.14 & -0.14 & -0.13 & -0.12 & 0.14 \\
\hline Aristolochia paucinervis Pomel & S-P & E3 & 3.01 & 0.07 & 0.08 & 0.09 & 0.11 & 0.11 & 0.16 & -0.06 & -0.16 & -0.16 & -0.12 & -0.10 & -0.10 & -0.19 & 0.01 & -0.18 & 0.08 \\
\hline $\begin{array}{l}\text { Quercus broteroi (Cout.) Rivas } \\
\text { Mart. }\end{array}$ & Q-I & E1 & 2.91 & 0.29 & 0.18 & 0.23 & 0.32 & 0.25 & 0.18 & 0.23 & -0.18 & -0.18 & -0.07 & -0.01 & 0.00 & -0.03 & 0.16 & -0.19 & 0.20 \\
\hline $\begin{array}{l}\text { Acer monspessulanum subsp. } \\
\text { monspessulanum L. }\end{array}$ & Q-F & E2 & 2.89 & 0.20 & 0.10 & 0.14 & 0.22 & 0.16 & -0.03 & 0.17 & 0.03 & 0.03 & 0.02 & 0.11 & 0.10 & 0.12 & 0.14 & 0.01 & 0.12 \\
\hline Primula acaulis (L.) L. & Q-F & E3 & 2.87 & -0.18 & -0.25 & -0.18 & -0.12 & -0.17 & -0.42 & 0.26 & 0.42 & 0.42 & 0.45 & 0.47 & 0.49 & 0.50 & 0.16 & 0.42 & -0.23 \\
\hline $\begin{array}{l}\text { Teucrium scorodonia subsp. } \\
\text { scorodonia } \mathrm{L} \text {. }\end{array}$ & Q-I & E3 & 2.83 & 0.27 & 0.24 & 0.32 & 0.25 & 0.34 & 0.16 & 0.20 & -0.16 & -0.16 & -0.09 & -0.08 & 0.08 & -0.02 & 0.06 & -0.19 & 0.32 \\
\hline Sorbus aria (L.) Crantz & $\mathrm{O}-\mathrm{F}$ & E2 & 2. & -0.24 & -0.29 & -0.31 & -0.27 & -0.30 & -0.02 & -0.13 & 0.0 & 0.0 & 0.05 & 0 & 0.00 & 0.02 & 0.08 & 0.05 & -0.28 \\
\hline Ilex aquifolium $\mathrm{L}$. & Q-F & E2 & 2.76 & -0.11 & -0.19 & -0.11 & -0.10 & -0.10 & -0.29 & 0.23 & 0.29 & 0.29 & 0.37 & 0.38 & 0.40 & 0.40 & 0.09 & 0.30 & -0.13 \\
\hline $\begin{array}{l}\text { Luzula forsteri subsp. forsteri (Sm.) } \\
\text { DC. in Lam. \& DC. }\end{array}$ & Q-I & E3 & 2.72 & 0.12 & -0.04 & 0.06 & 0.10 & 0.10 & 0.25 & -0.06 & -0.25 & -0.25 & -0.04 & -0.02 & 0.07 & -0.09 & 0.21 & -0.25 & 0.05 \\
\hline Erica arborea $\mathrm{L}$. & Q- & E2 & 2 & 0.3 & 0. & 0.3 & 0.29 & 0.32 & 0.0 & 0.21 & -0.06 & -0.06 & -0.06 & -0.03 & 0.08 & 0.07 & 0.10 & -0.09 & 0.29 \\
\hline Doronicum plantagineum $\mathrm{L}$. & Q-I & E3 & 2.67 & 0.03 & -0.03 & -0.04 & 0.01 & -0.03 & 0.18 & -0.06 & -0.18 & -0.18 & -0.20 & -0.13 & -0.15 & -0.23 & 0.07 & -0.17 & -0.03 \\
\hline $\begin{array}{l}\text { Quercus coccifera subsp. coccifera } \\
\text { L. }\end{array}$ & Q-F & E2 & 2.61 & 0.07 & 0.25 & 0.24 & 0.11 & 0.22 & 0.27 & 0.17 & -0.27 & -0.27 & -0.04 & -0.07 & -0.13 & -0.23 & -0.21 & -0.28 & 0.23 \\
\hline Pistacia terebinthus $\mathrm{L}$. & S- & E2 & 2 & 0.20 & 0. & 0. & 0.22 & 0. & 0.0 & 0.16 & -0.02 & -0.02 & 0.1 & 0.08 & 0.05 & 0.02 & 0.06 & -0.04 & 0.16 \\
\hline Ruscus aculeatus $\mathrm{L}$. & Q-I & E & 2.38 & 0.23 & 0.2 & 0.2 & 0.26 & 0.28 & 0.0 & 0.1 & -0.03 & -0.03 & -0.06 & -0.04 & 0.07 & 0.04 & -0.01 & & 0.26 \\
\hline Holcus mollis subs & $\mathrm{Q}-\mathrm{F}$ & E3 & 2.2 & 0.0 & 0.1 & 0.1 & -0.04 & 0.1 & 0 . & 0.05 & -0.16 & -0.16 & 0.0 & -0.05 & -0.05 & -0.11 & -0.20 & -0.17 & 0.19 \\
\hline Pistacia lentiscus L. & Q-I & E2 & 2.20 & 0.12 & 0.12 & 0.12 & 0.13 & 0.13 & 0.09 & 0.05 & -0.09 & -0.09 & -0.04 & 0.04 & -0.02 & -0.09 & 0.01 & -0.10 & 0.12 \\
\hline $\begin{array}{l}\text { Piptatherum paradoxum (L.) P. } \\
\text { Beauv. }\end{array}$ & Q-I & E3 & 2.20 & -0.04 & -0.06 & -0.05 & -0.04 & -0.04 & -0.17 & 0.14 & 0.17 & 0.17 & 0.15 & 0.20 & 0.17 & 0.17 & 0.05 & 0.17 & -0.06 \\
\hline
\end{tabular}


Table 5. Cont

\begin{tabular}{|c|c|c|c|c|c|c|c|c|c|c|c|c|c|c|c|c|c|c|c|}
\hline Species & $\begin{array}{c}\text { Phytosociological } \\
\text { Class }\end{array}$ & Stratum & IVI & $\operatorname{Tmax}$ & Tmin & $\mathrm{Tp}$ & PEs & PE & Iar & Id & $\mathrm{IH}$ & Ioe & Ios1 & Ios2 & Ios3 & Ios4 & Ic & Io & Itc \\
\hline Carex distachya Desf. & Q-I & E3 & 2.17 & 0.26 & 0.14 & 0.20 & 0.28 & 0.22 & 0.17 & 0.02 & -0.17 & -0.17 & -0.06 & -0.08 & 0.01 & -0.08 & 0.19 & -0.18 & 0.17 \\
\hline Phillyrea angustifolia $\mathrm{L}$. & $\mathrm{Q}-\mathrm{F}$ & E2 & 2.13 & 0.41 & 0.34 & 0.38 & 0.43 & 0.40 & 0.13 & 0.21 & -0.13 & -0.13 & -0.17 & -0.15 & 0.01 & -0.05 & 0.11 & -0.17 & 0.36 \\
\hline $\begin{array}{c}\text { Olea europaea var. sylvestris (Mill.) } \\
\text { Lehr }\end{array}$ & Q-I & E2 & 1.85 & 0.09 & 0.22 & 0.22 & 0.11 & 0.21 & 0.16 & 0.15 & -0.16 & -0.16 & -0.12 & -0.19 & -0.18 & -0.20 & -0.15 & -0.18 & 0.22 \\
\hline $\begin{array}{l}\text { Juniperus oxycedrus subsp. } \\
\text { oxycedrus L. }\end{array}$ & S-P & E2 & 1.84 & 0.18 & 0.10 & 0.15 & 0.22 & 0.17 & 0.05 & 0.16 & -0.05 & -0.05 & 0.08 & 0.15 & 0.10 & 0.09 & 0.14 & -0.06 & 0.12 \\
\hline $\begin{array}{l}\text { Paeonia officinalis subsp. } \\
\text { microcarpa (Boiss. \& Reut.) } \\
\text { Nyman }\end{array}$ & Q-I & E3 & 1.81 & -0.23 & -0.33 & -0.27 & -0.20 & -0.26 & -0.23 & 0.07 & 0.23 & 0.23 & 0.34 & 0.36 & 0.35 & 0.35 & 0.21 & 0.26 & -0.31 \\
\hline $\begin{array}{l}\text { Rhamnus alaternus subsp. } \\
\text { alaternus } \mathrm{L} \text {. }\end{array}$ & Q-I & E2 & 1.79 & 0.09 & 0.15 & 0.15 & 0.11 & 0.15 & 0.18 & 0.18 & -0.18 & -0.18 & 0.01 & 0.01 & -0.05 & -0.13 & -0.11 & -0.19 & 0.15 \\
\hline Rosa sicula Tratt. & Q-I & E3 & 1.76 & -0.19 & -0.16 & -0.23 & -0.21 & -0.23 & -0.02 & -0.14 & 0.02 & 0.02 & -0.03 & -0.07 & -0.04 & -0.04 & -0.02 & 0.05 & -0.20 \\
\hline $\begin{array}{c}\text { Bunium macuca subsp. macuca } \\
\text { Boiss. }\end{array}$ & S-P & E3 & 1.74 & -0.10 & -0.04 & -0.10 & -0.10 & -0.11 & 0.09 & -0.13 & -0.09 & -0.09 & -0.09 & -0.13 & -0.10 & -0.12 & -0.07 & -0.07 & -0.08 \\
\hline $\begin{array}{l}\text { Fraxinus angustifolia subsp. } \\
\text { angustifolia Vahl }\end{array}$ & Q-I & E1 & 1.71 & 0.00 & 0.00 & -0.01 & 0.01 & 0.01 & -0.11 & 0.13 & 0.11 & 0.11 & 0.09 & 0.13 & 0.13 & 0.11 & 0.05 & 0.10 & -0.01 \\
\hline Smilax aspera L. & S-P & E2 & 1.69 & 0.12 & 0.23 & 0.22 & 0.13 & 0.21 & 0.02 & 0.12 & -0.02 & -0.02 & -0.13 & -0.14 & -0.14 & -0.10 & -0.17 & -0.05 & 0.22 \\
\hline Melica uniflora Retz. & Q-F & E3 & 1.65 & 0.15 & -0.14 & 0.02 & -0.15 & 0.04 & 0.05 & 0.15 & -0.05 & -0.05 & 0.15 & 0.14 & 0.15 & 0.10 & 0.15 & -0.03 & 0.12 \\
\hline $\begin{array}{l}\text { Viola sylvestris subsp. sylvestris } \\
\text { Lam. }\end{array}$ & Q-F & E3 & 1.62 & -0.09 & -0.10 & -0.08 & -0.05 & -0.07 & -0.21 & 0.12 & 0.21 & 0.21 & 0.22 & 0.22 & 0.22 & 0.23 & 0.04 & 0.21 & -0.09 \\
\hline Vincetoxicum nigrum (L.) Moench & S-P & $\mathrm{E}$ & 1.57 & 0.18 & 0.08 & 0.13 & 0.20 & 0.14 & 0.01 & 0.17 & -0.01 & -0.01 & 0.01 & 0.06 & 0.09 & 0.08 & 0.16 & -0.03 & 0.10 \\
\hline Euphorbia characias L. & $\mathrm{Q}-\mathrm{F}$ & E3 & 1.56 & -0.10 & -0.09 & -0.16 & -0.14 & -0.17 & 0.04 & -0.19 & -0.04 & -0.04 & -0.17 & -0.21 & -0.19 & -0.18 & -0.03 & -0.02 & -0.13 \\
\hline Sanicula europaea L. & $\mathrm{Q}-\mathrm{F}$ & E3 & 1.55 & 0.05 & -0.16 & -0.03 & -0.09 & -0.02 & -0.01 & 0.14 & 0.01 & 0.01 & 0.18 & 0.20 & 0.19 & 0.14 & 0.17 & 0.02 & 0.00 \\
\hline $\begin{array}{c}\text { Lathyrus linifolius (Reichard) } \\
\text { Bässler }\end{array}$ & S-P & E3 & 1.44 & 0.01 & -0.11 & -0.06 & 0.01 & -0.02 & 0.10 & 0.00 & -0.10 & -0.10 & 0.14 & 0.12 & 0.14 & -0.01 & 0.12 & -0.10 & -0.06 \\
\hline Asparagus acutifolius L. & Q-I & E3 & 1.42 & 0.18 & 0.20 & 0.20 & 0.18 & 0.20 & 0.03 & 0.10 & -0.03 & -0.03 & -0.14 & -0.09 & -0.04 & -0.06 & 0.00 & -0.05 & 0.20 \\
\hline Asplenium onopteris $\mathrm{L}$. & Q-I & E3 & 1.41 & 0.22 & 0.22 & 0.24 & 0.24 & 0.25 & -0.02 & 0.04 & 0.02 & 0.02 & -0.15 & -0.11 & 0.06 & 0.06 & 0.05 & -0.01 & 0.23 \\
\hline $\begin{array}{c}\text { Conopodium pyrenaeum (Loisel.) } \\
\text { Miégev. }\end{array}$ & Q-I & E3 & 1.40 & -0.06 & -0.18 & -0.13 & -0.03 & -0.11 & -0.01 & -0.06 & 0.01 & 0.01 & 0.14 & 0.13 & 0.12 & 0.05 & 0.22 & 0.04 & -0.16 \\
\hline Taxus baccata $\mathrm{L}$. & O-F & E1 & 1.39 & -0.05 & -0.16 & -0.09 & -0.10 & -0.09 & -0.08 & 0.02 & 0.08 & 0.08 & 0.16 & 0.19 & 0.17 & 0.13 & 0.10 & 0.11 & -0.08 \\
\hline Sanguisorba hybrida (L.) Font Quer & $\mathrm{Q}-\mathrm{F}$ & $\mathrm{E}$ & 1.38 & 0.06 & 0.17 & 0.18 & 0.08 & 0.17 & 0.03 & 0.12 & -0.03 & -0.03 & -0.13 & -0.15 & -0.09 & -0.09 & -0.11 & -0.05 & 0.18 \\
\hline Paeonia coriacea Boiss. & Q-F & $\mathrm{E}$ & 135 & -0.07 & -0.04 & -0.10 & -0.10 & -0.11 & 0. & -0.17 & -0.08 & -0.08 & -0.21 & -0.20 & -0.25 & -0.22 & -0.04 & -0.06 & -0.08 \\
\hline Lonicera implexa Aiton & Q-I & $\mathrm{E}$ & 1.32 & 0.15 & 0.17 & 0.16 & 0.16 & 0.16 & 0.12 & 0.10 & -0.12 & -0.12 & -0.10 & -0.04 & -0.08 & -0.13 & -0.07 & -0.14 & 0.16 \\
\hline Hepatica nobil & Q-I & L & 1.29 & 0. & -0.14 & -0.02 & -0.13 & -0.01 & -0.03 & 0.16 & 0.03 & 0.0 & 0.18 & 0.18 & 0.19 & 0.15 & 0.06 & 0.04 & 0.03 \\
\hline Genista tournefortii Spach & $\widehat{Q}-\mathrm{I}$ & E3 & 1.28 & 0.25 & 0.17 & 0.20 & 0.24 & 0.22 & 0.08 & 0.12 & -0.08 & -0.08 & -0.13 & -0.10 & 0.02 & -0.04 & 0.12 & -0.10 & 0.19 \\
\hline $\begin{array}{l}\text { Asparagus aphyllus subsp. } \\
\text { aphyllus } \mathrm{L} \text {. }\end{array}$ & Q-F & E3 & 1.27 & 0.04 & 0.16 & 0.16 & 0.06 & 0.16 & 0.14 & 0.10 & -0.14 & -0.14 & -0.10 & -0.17 & -0.16 & -0.16 & -0.16 & -0.15 & 0.16 \\
\hline Potentilla sterilis (L.) Garcke & Q-F & $\mathrm{E}^{3}$ & 1. & 0 . & 0. & 0 & 0.0 & 0.0 & -0.13 & -0.09 & 0. & 0.1 & -0.06 & -0.12 & -0.11 & 0.01 & -0.10 & 0.12 & 0.12 \\
\hline Pyrus bourgaeana Decne. & Q-F & E2 & 1.21 & 0.13 & 0.16 & 0.16 & 0.14 & 0.16 & 0.10 & 0.09 & -0.10 & -0.10 & -0.09 & -0.08 & -0.05 & -0.11 & -0.05 & -0.11 & 0.16 \\
\hline Genista falcata Brot. & $\mathrm{Q}-\mathrm{F}$ & E3 & 1.18 & -0.06 & -0.11 & -0.07 & -0.02 & -0.05 & 0.12 & -0.08 & -0.12 & -0.12 & 0.10 & 0.12 & 0.14 & 0.03 & 0.07 & -0.12 & -0.10 \\
\hline $\begin{array}{l}\text { Dryopteris affinis subsp. affinis } \\
\text { (Lowe) Fraser-Jenk. }\end{array}$ & Q-F & E3 & 1.06 & 0.03 & -0.13 & -0.06 & -0.13 & -0.05 & -0.03 & 0.11 & 0.03 & 0.03 & 0.12 & 0.14 & 0.15 & 0.11 & 0.04 & 0.04 & 0.00 \\
\hline Ulmus glabra Huds. & Q-F & E1 & 1.03 & -0.04 & -0.08 & -0.06 & -0.03 & -0.05 & -0.10 & 0.07 & 0.10 & 0.10 & 0.13 & 0.1 & 0.14 & 0.13 & 0.08 & 0.10 & -0.07 \\
\hline Euphorbia hyberna L. & Q-F & E3 & 1.03 & -0.09 & -0.05 & -0.07 & -0.08 & -0.06 & -0.14 & 0.01 & 0.14 & 0.14 & 0.04 & 0.05 & 0.11 & 0.14 & -0.10 & 0.14 & -0.06 \\
\hline
\end{tabular}


Table 5. Cont

\begin{tabular}{|c|c|c|c|c|c|c|c|c|c|c|c|c|c|c|c|c|c|c|c|}
\hline Species & $\begin{array}{c}\text { Phytosociological } \\
\text { Class }\end{array}$ & Stratum & IVI & $\operatorname{Tmax}$ & Tmin & $\mathrm{Tp}$ & PEs & PE & Iar & Id & IH & Ioe & Ios1 & Ios2 & Ios3 & Ios4 & Ic & Io & Itc \\
\hline Veronica officinalis $\mathrm{L}$. & Q-F & E3 & 1.02 & 0.02 & -0.16 & -0.05 & -0.14 & -0.04 & -0.08 & 0.16 & 0.08 & 0.08 & 0.21 & 0.20 & 0.21 & 0.18 & 0.10 & 0.09 & 0.00 \\
\hline Bupleurum rigidum subsp. & Q-F & E3 & 1.01 & 0.13 & 0.11 & 0.11 & 0.11 & 0.11 & 0.02 & 0.14 & -0.02 & -0.02 & -0.09 & -0.03 & -0.03 & -0.05 & 0.00 & -0.03 & 0.11 \\
\hline Senecio lopezii Boiss. & S-P & E3 & 0.98 & 0.10 & 0.15 & 0.13 & 0.07 & 0.11 & -0.12 & -0.11 & 0.12 & 0.12 & -0.06 & -0.13 & -0.13 & -0.03 & -0.12 & 0.10 & 0.14 \\
\hline Carex pendula Huds. & Q-I & E3 & 0.98 & -0.05 & -0.06 & -0.05 & -0.05 & -0.05 & -0.11 & 0.11 & 0.11 & 0.11 & 0.10 & 0.11 & 0.12 & 0.12 & 0.02 & 0.11 & -0.06 \\
\hline Moehringia pentandra J. Gay & S-P & E4 & 0.97 & 0.16 & 0.08 & 0.12 & 0.19 & 0.14 & 0.16 & 0.12 & -0.16 & -0.16 & -0.04 & 0.01 & 0.01 & -0.04 & 0.12 & -0.16 & 0.10 \\
\hline $\begin{array}{c}\text { Ajuga x_rotundifolia Willk. \& } \\
\text { Cutanda ex Willk. }\end{array}$ & S-P & E3 & 0.92 & 0.12 & -0.12 & 0.06 & -0.12 & 0.10 & 0.09 & 0.12 & -0.09 & -0.09 & 0.12 & 0.12 & 0.12 & 0.07 & 0.12 & -0.07 & 0.12 \\
\hline Phillyrea latifolia subsp. latifolia $\mathrm{L}$. & Q-F & E2 & 0.91 & 0.15 & 0.10 & 0.12 & 0.16 & 0.13 & 0.02 & 0.11 & -0.02 & -0.02 & -0.04 & 0.03 & 0.03 & 0.03 & 0.06 & -0.04 & 0.11 \\
\hline $\begin{array}{l}\text { Juniperus phoenicea subsp. } \\
\text { phoenicea L. }\end{array}$ & Q-I & E2 & 0.86 & 0.05 & 0.13 & 0.11 & 0.06 & 0.10 & 0.14 & 0.06 & -0.14 & -0.14 & 0.07 & 0.07 & -0.02 & -0.10 & -0.11 & -0.14 & 0.11 \\
\hline $\begin{array}{l}\text { Clematis campaniflora Brot. } \\
\text { Carex sylvatica Huds. }\end{array}$ & $\begin{array}{l}\text { Q-I } \\
\text { Q-F }\end{array}$ & $\begin{array}{l}\text { E2 } \\
\text { E3 }\end{array}$ & $\begin{array}{l}0.83 \\
0.82\end{array}$ & $\begin{array}{c}0.12 \\
-0.06\end{array}$ & $\begin{array}{c}0.11 \\
-0.09\end{array}$ & $\begin{array}{l}0.11 \\
-0.08\end{array}$ & $\begin{array}{c}0.10 \\
-0.06\end{array}$ & $\begin{array}{c}0.11 \\
-0.08\end{array}$ & 0.01 & $\begin{array}{l}0.10 \\
0.11\end{array}$ & $\begin{array}{c}-0.01 \\
0.11\end{array}$ & $\begin{array}{c}-0.01 \\
0.11\end{array}$ & $\begin{array}{c}-0.11 \\
0.12\end{array}$ & $\begin{array}{c}-0.03 \\
0.13\end{array}$ & $\begin{array}{c}-0.04 \\
0.14\end{array}$ & $\begin{array}{c}-0.05 \\
0.14\end{array}$ & $\begin{array}{c}-0.03 \\
0.06\end{array}$ & $\begin{array}{c}-0.02 \\
0.12\end{array}$ & $\begin{array}{c}0.11 \\
-0.09\end{array}$ \\
\hline $\begin{array}{c}\text { Carex sylvatica Huds. } \\
\text { Epipactis microphylla (Ehrh.) Sw. }\end{array}$ & $\begin{array}{l}\text { Q-F } \\
\text { Q-F }\end{array}$ & $\begin{array}{l}\mathrm{E} 3 \\
\mathrm{E} 3\end{array}$ & $\begin{array}{l}0.82 \\
0.71\end{array}$ & $\begin{array}{l}-0.06 \\
-0.05\end{array}$ & $\begin{array}{l}-0.09 \\
-0.09\end{array}$ & $\begin{array}{l}-0.08 \\
-0.07\end{array}$ & $\begin{array}{l}-0.06 \\
-0.04\end{array}$ & $\begin{array}{l}-0.08 \\
-0.06\end{array}$ & $\begin{array}{l}-0.11 \\
-0.11\end{array}$ & $\begin{array}{l}0.11 \\
0.10\end{array}$ & 0.11 & $\begin{array}{l}0.11 \\
0.11\end{array}$ & $\begin{array}{l}0.12 \\
0.13\end{array}$ & $\begin{array}{l}0.13 \\
0.15\end{array}$ & $\begin{array}{l}0.14 \\
0.14\end{array}$ & $\begin{array}{l}0.14 \\
0.14\end{array}$ & $\begin{array}{l}0.00 \\
0.08\end{array}$ & $\begin{array}{l}0.12 \\
0.11\end{array}$ & $\begin{array}{l}-0.09 \\
-0.08\end{array}$ \\
\hline Anemone palmata $\mathrm{L}$. & Q-I & E3 & 0.69 & 0.13 & 0.09 & 0.10 & 0.14 & 0.11 & 0.10 & 0.12 & -0.10 & -0.10 & -0.04 & -0.02 & -0.03 & -0.06 & 0.06 & -0.12 & 0.10 \\
\hline Viola suavis M. Bieb. & $\mathrm{Q}-\mathrm{F}$ & E3 & 0.69 & -0.06 & -0.12 & -0.09 & -0.05 & -0.09 & -0.09 & 0.02 & 0.09 & 0.09 & 0.13 & 0.15 & 0.13 & 0.12 & 0.13 & 0.10 & -0.11 \\
\hline Iris foetidissima $\mathrm{L}$. & S-P & E3 & 0.66 & -0.08 & -0.12 & -0.10 & -0.08 & -0.10 & -0.12 & 0.10 & 0.12 & 0.12 & 0.12 & 0.12 & 0.13 & 0.14 & 0.07 & 0.13 & -0.11 \\
\hline
\end{tabular}

Significant values to $95 \%$ are shown in grey. Taxa that do not show any significant correlation were eliminated. The taxa are listed in order of importance (IVI). The phytosociological adscription of the taxa is also shown: Q-F (Querco roboris-Fagetea sylvaticae), Q-I (Quercetea ilicis), S-P (Salici purpureae-Populetea nigrae). 


\subsubsection{Canonical Correlation Analysis (CCA)}

The bioclimatic variables and the taxa with the highest abundance levels in the 421 relevés studied were analysed by canonical correspondence analysis. Here, $79.33 \%$ of the variability between the bioclimatic data and the abundance of Querco roboris-Fagetea sylvaticae and Quercetea ilicis species is explained on the first two axes (Figure 3). The horizontal axis represents $44.97 \%$ of the variability and can be understood as a temperature gradient. The bioclimatic variables are PEs, Iar, Pe, Tmax, Tmin, Tmed and Itc, shown in the lower left quadrant. The species with the highest correlation with these bioclimatic variables are Smilax aspera, Quercus coccifera subsp. coccifera, and Juniperus phoenicea subsp. phoenicea, which have a preference for warm bioclimates. These taxa do not grow in humid biotopes or with high values of Io or Ioe, except on sites with a steeply sloping topography or soil conditions that do not allow the use of rainwater (edaphoxerophilous) $[1,4,20,76]$. Tamus communis, Arbutus unedo, Daphne gnidium, Paeonia broteri, and Erica arborea are correlated to a lesser extent with these variables and show no correlations with Itc, Tmax, or Tmin gradients. These taxa have a broad distribution, and bioclimatic variables do not significantly influence their occurrence or abundance [77]. There is also a smaller group which includes species with high values of PE or Iar (Figure 2), such as Quercus rotundifolia, Quercus broteroi, Quercus coccifera subsp. Coccifera, and Phillyrea angustifolia.

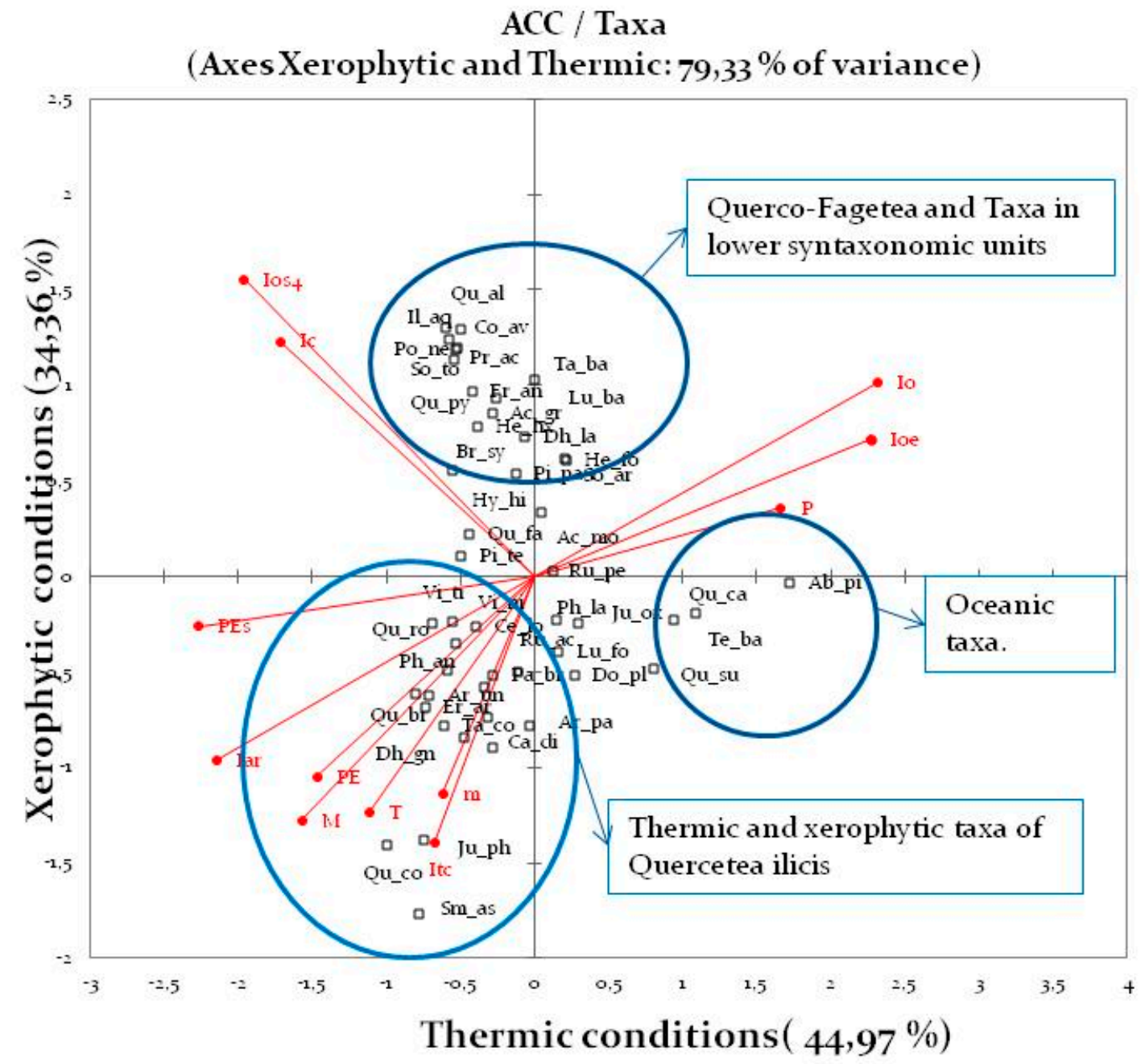

Figure 3. Canonical correlation analysis (CCA) between the bioclimatic variables and the taxa in the relevés studied. 
It is worth mentioning the taxa that correlate very positively with precipitation (P), Ioe, and Io in the lower right quadrant and away from biotopes with a high continentality index (Ic). It can therefore be said that taxa such as Abies pinsapo, Teucrium scorodonia subsp. baeticum, Quercus suber var. suber, and Quercus canariensis are typical of humid and oceanic habitats where Ic values are low. Doronicum plantagineum and Luzula forsteri subsp. baetica also correlate with high Itc values.

The vertical axis explains $34.36 \%$ of the variability (Figure 3). It can be seen in the upper left quadrant that Ios4 and Ic more positively correlate with the vertical axis, and negatively with the horizontal axis. The taxa positively correlated with Ios4 and Ic are Quercus pyrenaica, Acer opalus subsp. granatense, Quercus faginea subsp. alpestris, Hedera helix subsp. helix, Ilex aquifolium, Primula acaulis, Corylus avellana, Sorbus torminalis, Poa nemoralis and Brachypodium sylvaticum. In contrast, Hyacinthoides hispanica, Arenaria montana subsp. montana, Quercus faginea subsp. faginea, and Acer monspessulanum subsp. monspessulanum show lower correlations. It is also worth noting the correlations between Io, Ioe and $P$ and the occurrence and abundance of other characteristic taxa of Querco roboris-Fagetea sylvaticae such as Helleborus foetidus, Daphne laureola subsp. latifolia, Sorbus aria, Taxus baccata, and Luzula forsteri subsp. baetica

The location of all the above-mentioned taxa (far from the high values of variables related to thermicity, xericity, and evapotranspiration such as PE, PEs, Iar, Tmax, Tmin, and Itc) supports the adscription of these species to the classes Querco robori-Fagetea sylvaticae or Salici purpureae-Populetea nigrae in the phytosociological plant community classification model $[70,71]$. However, thermophilous and xerophilous species and species that tolerate or have a preference for biotopes with high levels of PE or PEs are typical of Quercetea ilicis.

\subsection{ANOVA Analysis of Introgression of Quercetea ilicis Species in Deciduous Forests}

Table 6 shows an analysis of the correlation between the bioclimatic variables and the frequency of occurrence of typical species of dry environments with the introgression of taxa that are characteristic of the dry or warm environments typical of the Mediterranean macrobioclimate. This correlation is statistically significant with Tmin, Tp, PEs, PE, Iar, Ioe, $\operatorname{Ios}_{1}, \operatorname{Ios}_{2}, \operatorname{Ios}_{3}, \operatorname{Ios}_{4}$, and Io; that is, with the bioclimatic indices related to temperature, soil moisture loss, and water availability.

Table 6. Analysis of variance between the bioclimatic variables and the frequency of occurrence of Querceta ilicis taxa. Statistically significant values at 95\% confidence level are shown in bold.

\begin{tabular}{cccccccc}
\hline $\begin{array}{c}\text { Climate } \\
\text { Variable }\end{array}$ & $\mathbf{R}^{2}$ & $\mathbf{F}$ & $\begin{array}{c}\text { Pr }>\mathbf{F} \\
(\boldsymbol{p} \text {-Value })\end{array}$ & $\begin{array}{c}\text { Climate } \\
\text { Variable }\end{array}$ & $\mathbf{R}^{2}$ & $\mathbf{F}$ & $\begin{array}{c}\text { Pr }>\mathbf{F} \\
(\boldsymbol{p} \text {-Value })\end{array}$ \\
\hline Tmax & 0.0018 & 0.1569 & 0.6930 & Ioe & 0.1983 & 21.2673 & $<0.0001$ \\
Tmin & 0.5960 & 126.8766 & $<0.0001$ & Ios1 & 0.2591 & 30.0685 & $<0.0001$ \\
Tp & 0.5550 & 107.2388 & $<0.0001$ & Ios2 & 0.3395 & 44.1971 & $<0.0001$ \\
PEs & 0.4093 & 59.5860 & $<0.0001$ & Ios3 & 0.4922 & 83.3669 & $<0.0001$ \\
PE & 0.5089 & 89.1102 & $<0.0001$ & Ios4 & 0.4068 & 58.9869 & $<0.0001$ \\
Iar & 0.1842 & 19.4198 & $<0.0001$ & Ic & 0.0668 & 6.1594 & 0.0150 \\
Id & 0.0052 & 0.4524 & 0.5030 & Io & 0.2589 & 30.0473 & $<0.0001$ \\
IH & 0.1983 & 21.2673 & $<0.0001$ & Itc & 0.0329 & 2.9248 & 0.0908 \\
\hline
\end{tabular}

The regression model (Table 6) reveals a significant positive correlation $\left(R^{2}=0.596, p\right.$-value $\left.<0.0001\right)$ between the frequency of Quercetea ilicis species and increases in Tmin. A similar pattern has been noted with positive temperature (Tp) with values of $R^{2}=0.555$ and a $p$-value $<0.0001$. These results imply that the occurrence of taxa typical of the Mediterranean macrobioclimate is also higher when $\mathrm{Tp}$ is higher. PEs $\left(R^{2}=0.4093, p\right.$-value $\left.<0.0001\right)$ and PE $\left(R^{2}=0.5089, p\right.$-value $\left.<0.0001\right)$ also show significant positive correlations. This result highlights the association between the frequency of occurrence of species growing in xeric environments and the high levels of annual and summer evapotranspiration typical of the Mediterranean sclerophyllous formations of Quercetea ilicis. The aridity index (Iar) 
also has a significant positive correlation with the occurrence of Quercetea ilicis species $\left(R^{2}=0.1842\right.$, $p$-value $<0.0001)$.

Conversely, Ioe is negatively correlated $\left(R^{2}=0.1983, p\right.$-value $\left.<0.0001\right)$ with the occurrence of xerophytic species, signifying a higher number of species of Querco roboris-Fagetea sylvaticae, and that Quercetea ilicis taxa do not therefore appear in purely deciduous forests. Ios1 $\left(R^{2}=0.2591\right.$, $p$-value $<0.0001)$; Ios2 $\left(R^{2}=0.395, p\right.$-value $\left.<0.0001\right)$; Ios3 $\left(R^{2}=0.4922, p\right.$-value $\left.<0.0001\right)$; Ios4 $\left(R^{2}=0.4068, p\right.$-value $\left.<0.0001\right) ; \mathrm{IH}\left(\mathrm{R}^{2}=0.1983, p\right.$-value $\left.<0.0001\right)$; and Io $\left(\mathrm{R}^{2}=0.2589, p\right.$-value $\left.<0.0001\right)$ show a similar behaviour with regard to the occurrence of typical species of Quercetea ilicis. This suggests that in sampling points with higher values of these bioclimatic variables, the occurrence of species typical of drier environments in relation to species characteristic of deciduous forests is less frequent than in sampling points with lower values of these bioclimatic variables.

\subsection{Frequency of Occurrence and Average Cover in the Floristic Composition of Each Plant Community}

The analysis of the plant community known as Adenocarpo decorticantis-Quercetum pyrenaicae, (AdQp) corresponding to subhumid-humid meso-supramediterranean formations, whose distribution area is limited to the Nevadensean and Almijarensean sector in the Baetic province (Figure 4a), reveal a majority composition of taxa characteristic of the deciduous forest (Table A1).
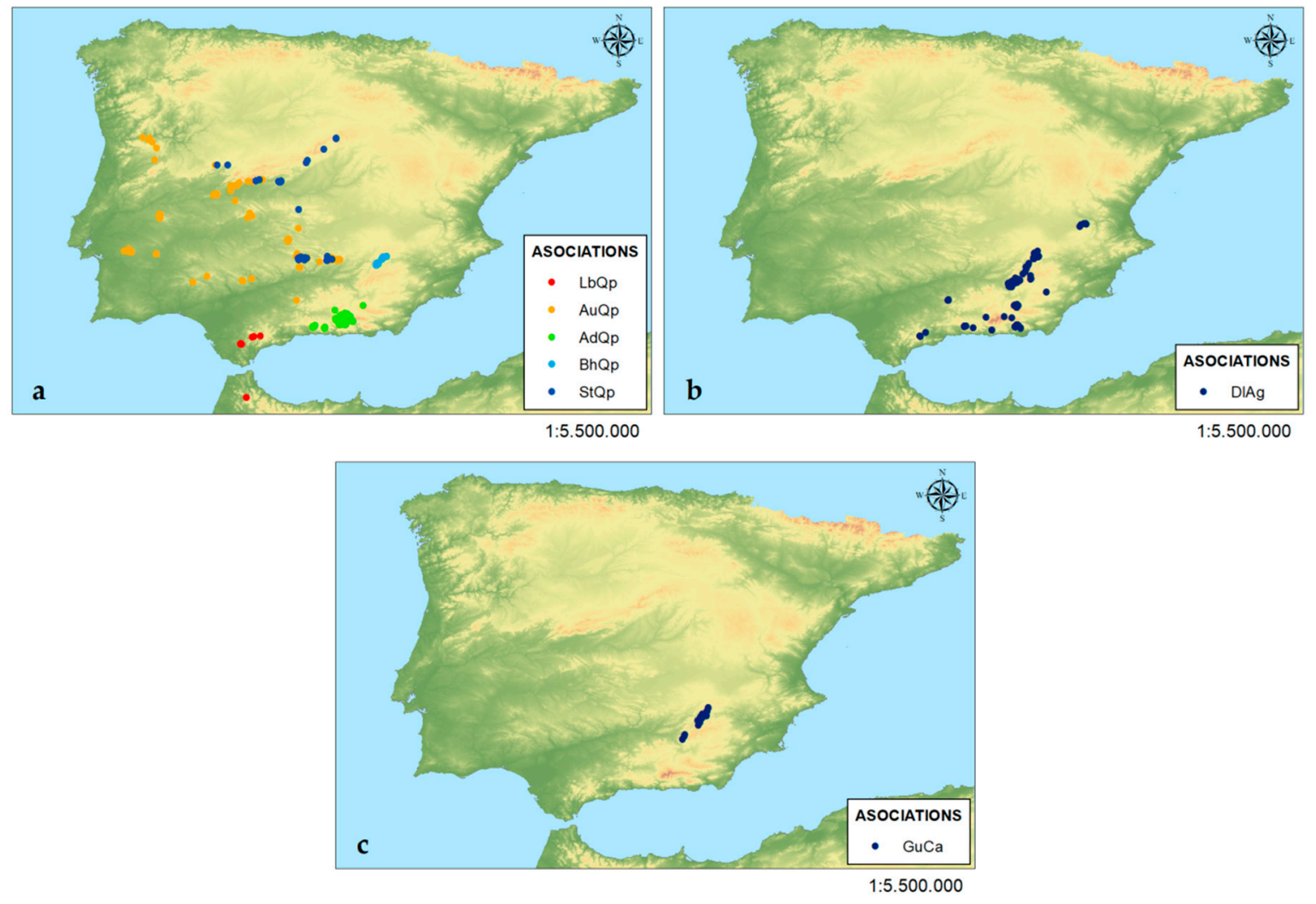

Figure 4. Distribution of deciduous forests in the southern-central part of the Iberian Peninsula: (a) distribution of formations dominated by Quercus pyrenaica; (b) distribution of formations dominated by Acer opalus subsp. granatense; (c) distribution of formations dominated by Corylus avellana.

It can also be seen in Table A1 that the community includes taxa typical of the Mediterranean forest in its floristic composition. It is worth highlighting the presence in the deciduous forest of tree species such as Quercus rotundifolia and Pistacia terebinthus which appear in $23 \%$ and $1.6 \%$ of the samplings, albeit with low cover and de stratum 2 on this formation.

The analysis of the vegetation in the Pyrenean oak forests known as Arbuto unedonis-Quercetum pyrenaicae, and corresponding to the subhumid-humid thermo-mesomediterranean forests of the 
Luso-Extremaduran province (Figure 4a), reveals a floristic composition typical of deciduous forests (Table A1). The introgression of taxa characteristic of the Mediterranean forest can be seen in Table A1. There is a significant presence of tree and shrub species with high presence indices such as Quercus suber (35.7\%), Quercus rotundifolia (33.6\%), Quercus broteroi (20.3\%), and Arbutus unedo (50.3\%), although Quercus broteroi and Quercus suber belong to forests in transition to strictly deciduous forests and are in stratum 1.

The analysis of the Luso-Extremaduran subhumid-humid supramediterranean Pyrenean oak forests, Sorbo torminalis-Quercetum pyrenaicae (Figure 4a), reveals the highest proportion of typical deciduous forest species in their composition, with a proportion of $52.17 \%$. The frequencies and average covers are shown in Table 4 , which shows that the only tree species with significant frequency and cover is Quercus broteroi, with a frequency of occurrence of $14 \%$ and a low average cover of $6.3 \%$.

The particularity of the Baetic Pyrenean oak forests on siliceous sandstones in the Utrillas facies in the Subbetic sector, the meso-supramediterranean Berberido australis-Quercetum pyrenaicae (Figure 4a) in a lower humid-upper humid ombroclimate, can be seen from its floristic composition, as shown in Table A1. The characteristic tree species of the Mediterranean forest, occurring with a high frequency although with sparse average cover, is Quercus rotundifolia, with a frequency of $33.3 \%$ and an average cover of $8.3 \%$, and Pinus pinaster subsp. pinaster, with a frequency of $22.2 \%$ and an average cover of $3.8 \%$.

The southernmost formations of Quercus pyrenaica, known as Luzulo baetici-Quercetum pyrenaicae, are characterised by their distribution in a humid-hyperhumid ombroclimate in the thermo-mesomediterranean belt (Figure 4a). These formations have a scarce representation and a limited distribution area, as demonstrated by the 10 samplings available. Their floristic composition is shown in Table A1. These Pyrenean oak forests reveal a very significant incorporation of typical species of more xeric Mediterranean forests, as demonstrated by the low proportion of characteristic deciduous forest species (30.7\%).

Figure $4 \mathrm{~b}$ shows an analysis of the floristic composition of the meso-supramediterranean maple woodlands growing on base-rich soils in the Betic province, Daphno latifoliae-Aceretum granatensis [32]. It includes a high diversity of phanerophytes, such as Acer opalus subsp. granatense, Quercus pyrenaica, Quercus faginea subsp. alpestris, Quercus faginea subsp. faginea, Ilex aquifolium, Corylus avellana, Sorbus torminalis, Sorbus aria, Acer monspessulanum subsp. monspessulanum, Buxus sempervirens, Taxus baccata, and Ulmus glabra. Hedera helix subsp. Helix and Tamus communis are in stratum 2 exclusively.

There are also lower-growing taxa in stratum 3 and 4 such as Helleborus foetidus, Daphne laureola subsp. latifolia, Primula acaulis, Polygonatum odoratum, Paeonia officinalis L. subsp. microcarpa, Cephalanthera longifolia, Viola sylvestris subsp. sylvestris, Primula veris, Conopodium pyrenaeum, Vinca minor L., and Epipactis microphylla. All these taxa are characteristic of Querco roboris-Fagetea sylvaticae.

The introgression of species typical of more xeric environments (Quercetea ilicis) is significant due to the presence of Quercus rotundifolia Lam., which has a frequency of occurrence of $36.6 \%$ and an average cover of $6.9 \%$, and Pinus pinaster subsp. pinaster, with a frequency of $6.3 \%$ and average cover of $37.5 \%$. Juniperus oxycedrus subsp. oxycedrus also has a high occurrence $(16.1 \%)$ and cover of $3.5 \%$. Quercus coccifera subsp. coccifera and Juniperus phoenicea subsp. phoenicea (occurrence of $5.4 \%$ and $6.3 \%$, respectively, and average covers of $11.3 \%$ and $2.9 \%$ ) complete the list of phanerophytes, which in other more xeric conditions establish plant communities with species typical of Querceta ilicis $[1,20,76,78,79]$, accounting for $40.98 \%$ of maple woodland species (Table 7 ).

Table 7. Frequency of occurrence of taxa by phytosociological class for each plant community studied.

\begin{tabular}{cccccccc}
\hline Phytosociological Class & AdQp & AuQp & BhQp & LbQp & StQp & DlAg & GuCa \\
\hline Querco roboris-Fagetea sylvaticae & 54.84 & 49.41 & 69.23 & 27.78 & 60.92 & 40.98 & 60.42 \\
Quercetea ilicis & 32.26 & 40 & 23.08 & 66.67 & 26.44 & 40.98 & 22.92 \\
Salici purpureae-Populetea nigrae & 12.9 & 10.59 & 7.692 & 5.556 & 12.64 & 18.03 & 16.67 \\
\hline
\end{tabular}


The occurrence of both native and naturalised gymnosperms is particularly significant, and is largely due to anthropic action in the form of fires or tree felling. These actions can cause erosion and soil loss, thus creating microbiotopes that favour the expansion of gymnosperms in times of abundant rains, which are typical of these locations $[20,76,80]$.

Hazelnuts are deciduous meso-supramediterranean formations located in ravines and shady and nemoral exposures, always in conditions of soil compensation within the Subbetic sector [21] (Figure 4c). These are the most humid formations, and are even sub-riparian in some sites. Their floristic composition (Table A1) includes particularly a high presence of Querco roboris-Fagetea sylvaticae taxa $(60.42 \%)$ (Table 7$)$. In addition to $16.67 \%$ of taxa characteristic of Salici purpureae-Populetea nigrae, the proportion of species typical of drier environments is the lowest in all the plant communities studied, with $22.92 \%$ (Table 7 ).

Among the species of Quercetea ilicis, it is especially worth noting the presence of Pistacia terebinthus, which occurs in $11.8 \%$ of the relevés and has an average cover of $3.8 \%$; Viburnum tinus., with a presence of $11.8 \%$ and an average cover of $4.4 \%$; and Phillyrea latifolia subsp. latifolia (occurrence $8.8 \%$ and cover $3.3 \%$ ). All these species are part of the stratum 2. It is significant that although these species are characteristic of Quercetea ilicis, as they have a preference for sites with certain soil moisture $[20,77,81]$.

\section{Discussion}

In view of these results, it is clear that the deciduous formations in the southern-central part of the Iberian Peninsula are nemoral redoubts where various taxa took refuge in times of glaciations, retreating from the cold weather [82-84]. These species are typical of the temperate macrobioclimate and Eurosiberian biogeography $[31,85]$ and are currently found in more humid areas beside streams, in enclosed valleys, or on shady slopes in mountain formations with a higher rainfall regime $[21,28,30]$. These formations are therefore surrounded and are quickly replaced by other sclerophyllous formations when the particular microclimatic, topographic and soil conditions disappear $[1,4,20,76]$.

The typical forest dynamic leads to regular gaps in the tree or shrub canopy, resulting in a temporary and local increase in water and moisture loss. These openings are quickly colonised by shrubs and lianoid species characteristic of drier environments in the deciduous forests located further south $[86,87]$, which are found in greater or lesser numbers at the limits of these deciduous reducts within the Mediterranean macrobioclimate $[21,24,30]$. The mesophytic conditions remain unaffected in the absence of further pressure and or any sudden changes in bioclimatic conditions, and species such as Arbutus unedo, Phillyrea latifolia subsp. latifolia, Viburnum tinus, and Rhamnus alaternus subsp. alaternus in the stratum 2 behave as nurse species to the typical taxa in the dynamic stages of the deciduous forest that grow or are typical in stratum 3, comprising a mixture of shrubs or microphanerophytes and thorny shrubs (Rhamno catharticae-Prunetea spinosae) [21,30,88,89].

This ability to regenerate the climactic forest mostly depends on the climatic and bioclimatic conditions represented in the different bioclimatic variables analysed in the present study. High values of variables such as PEs, PE, Iar, and Tmin are correlated with a lower occurrence of taxa typical of Querco roboris-Fagetea sylvaticae and a lower capacity of these species to germinate, become established, and attain maturity $[9,18,22,23]$. There are also important differences in the floristic composition of the different plant communities studied, and a latitudinal gradient can be seen in the frequency of typical deciduous forest species.

It can be observed that the Quercus pyrenaica forests located further north in the study area considered in this paper, designated Sorbo torminalis-Quercetum pyrenaicae, have the highest proportion of species typical of cold moist places (Table 5) with respect to the number of characteristic taxa of Quercetea ilicis, in a ratio of about 4:1. Conversely, the Quercus pyrenaica forests of Luzulo baeticae-Quercetum pyrenaicae are located more to the south, at the limit of the distribution area $[90,91]$ and with a proportion of characteristic species of 1:3 in favour of the typical taxa of Quercetea ilicis. This implies additional pressure and hence a greater likelihood of these forests disappearing in the future. These distinctions 
between the forests located further south and those situated further north point to the need for different conservation efforts.

Several researchers [4,92-94] have warned of future changes in climate and thus bioclimatology, with a generalised increase in temperature and particularly the polarisation of the rainy season to a single season or to a shorter period of the year. Although it is true that a certain increase in rainfall can be expected in summer periods in the southern Iberian Peninsula, this type of rainfall is convective and associated to torrential rains with heavy rainfall in a very short time [95], so it cannot be used as efficiently by the vegetation due to the decoupling of the cycles of growth and water availability in the Mediterranean climate $[96,97]$. This could imply consequences for species that are highly dependent for their development on high values of Ioe, $\operatorname{Ios}_{1}, \operatorname{Ios}_{2}$, $\operatorname{Ios}_{3}$, Ios 4 , Io, or IH variables. These taxa will gradually be replaced by species that are more tolerant to low values of these bioclimatic indices.

According to the Fifth Assessment Report (AR5) [98], the climate system is unequivocally warming, and many of the changes seen since the 1950s are unprecedented in either decades or millennia. The report also highlights the advance of desertification, rising temperatures and decreasing rainfall, to which the areas located in North Africa and the southern Mediterranean-including the southern-central part of the Iberian Peninsula-are especially vulnerable. Another factor to take into account is the past, present, and future anthropic pressure affecting these relict forests, from either past logging that allowed the establishment of more xerophytic species; sustainable forest exploitation and thus the elimination of replacement thickets; fires, especially in monospecific pine formations for crops or reforestation [76,99]; and even water use, which reduces the groundwater level, creating added pressure and a greater opportunity for colonisation by more xerophilous species.

The great heterogeneity of these deciduous forests must be considered when establishing and managing different conservation strategies in future. All these formations are listed in the Habitats Directive [100]. Quercus pyrenaica forests are described as 9320: Galician-Portuguese oak woods with Quercus robur and Quercus pyrenaica. Maple and hazelnut formations are classified as 9240 Quercus faginea and Quercus canariensis Iberian woods. This excessive synthesis in the description, interpretation, and the establishment of conservation strategies in the Habitats Directive can lead to deficiencies in the conservation of flora and habitats [101], as the singularities of each formation are not studied in detail. In addition, these plant communities form small microtopes in the southern Iberian Peninsula.

As has been pointed out in the results section, the introgression of typical Mediterranean forest species can displace the characteristic taxa of the deciduous forests if the conditions required for their establishment are maintained or increased. The floristic composition of the deciduous formations in the southern-central part of the Iberian Peninsula are highly vulnerable due to the predicted increase in the values of PE, Pes, Iar, and Tmin and the decrease in IH, Ioe and the ombrothermic indices (Io, Ios) $[4,102]$. This primarily benefits the replacement of more stenoic nemoral species and secondly the replacement of the tree species typical of Querco Fagetea by those of Quercetea ilicis.

The effect of PE on vegetation and the change in the floristic composition of forests can be observed in [103-105], which studies how closed forests generate high evapotranspiration levels which maintain the nemoral conditions and favour the establishment of Querco roboris-Fagetea sylvaticae taxa. This phenomenon, whereby a closed forest paradoxically has higher PE values than a grass forest [103], ensures the appropriate microclimatic conditions for the establishment of these taxa. Reference [105] determines a period of 19 years for the establishment of shrub vegetation, during which PE decreases until the bushes grow, the leaf area and root systems increase, and PE levels start to recover. It is therefore possible to differentiate the PE due to the tree canopy, whose moisture is retained by the leaf area, and the PE caused in environments devoid of vegetation, where water is not captured and used by deciduous vegetation.

Some studies take a more general approach to the question of how plant dynamics may change in response to global warming, rather than by projecting trends and plant typologies (e.g., mountain vegetation [104], closed forests [103], phreatophyte vegetation [105]). However, they fail to clarify how each of the structural components in the different plant formations interact, and thus their explanatory 
dynamics. For example, taxa linked to high values of Io, Ioe, and $\operatorname{Ios}_{1-4}$-and hence to high water availability-disappear when PE values are high, but they can nevertheless be found in closed forests where the PE is higher.

The main limitations of the present work can be addressed through further research to study the dynamic stages of deciduous vegetation and correlate them with patterns in the bioclimatic indices projected for the southern-central part of the Iberian Peninsula. Models must be created to investigate the relation between the vegetation structure and the bioclimatic parameters of both deciduous and marscecent forests, their relation as forests surrounding fully deciduous forests, and ultimately their relation with evergreen forests which are much better adapted to the Mediterranean climate.

\section{Conclusions}

One consequence of rising temperatures due to global warming is the increase in evapotranspiration, and summer evapotranspiration in deciduous forests in the southern Iberian Peninsula. This is a factor that can endanger the continuity of these species. The deciduous forest located further south could be the most vulnerable.

In cooler environments, Sorbo torminalis-Quercetum pyrenaicae, Berberido hispanicae-Quercetum pyrenaicae, and Adenocarpo decorticantis-Quercetum pyrenaicae have greater resilience. The water compensation of the temporihygrophilous forests of Daphno latifoliae-Aceretum granatensis and Geo urbani-Coryletum avellanae attenuates evapotranspiration and favours a more continuous moisture supply. Deforestation and fires cause increased evapotranspiration and lead to the establishment of flora of Quercetea ilicis. The opening of gaps in the canopy provokes the loss of nemoral conditions and limits the development of the taxa that are most vulnerable to increased evapotranspiration, which may disappear.

Future research will go toward generating structural and dynamic vegetation models using a range of bioclimatic variables and inter- and intraspecific interaction-both in regard to the different taxa in a plant community and the dynamics of relationship facilitation and competition interactions [106] - among forest plant communities in order to project predictive models of global warming scenarios. These can be then used to reveal the most vulnerable taxa to enable their conservation, predict which taxa will replace and occupy their ecological niche, and provide insights into forest structures in the future.

Author Contributions: Conceptualisation; J.C.P.F. and E.C.; methodology; J.C.P.F., A.C.-O., and S.d.R.; software; J.C.P.F.; data curation; C.M.M., R.Q.C., and S.d.R.; writing-original draft preparation, translate; J.C.P.F.; writing-review and editing; J.C.P.F., E.C., A.C.-O., C.M.M., G.S., R.Q.C., C.J.P.G., and S.d.R. supervision; J.C.P.F., E.C., G.S., and C.J.P.G.

Funding: This research received no external funding.

Acknowledgments: Pru Brooke Turner (MA Cantab.) for the English translation of this article and the anonymous reviewers who have improved our manuscript with their comments.

Conflicts of Interest: The authors declare no conflict of interest. 


\section{Appendix A}

Table A1. Floristic composition, frequency of occurrence and average cover of taxa in each deciduous forest plant community studied.

\begin{tabular}{|c|c|c|c|c|c|c|c|c|c|c|c|c|c|c|c|c|c|}
\hline \multirow{2}{*}{ Taxon } & \multirow{2}{*}{ IVI } & \multirow{2}{*}{$\begin{array}{l}\text { Phytosociological } \\
\text { Class }\end{array}$} & \multirow{2}{*}{ Stratum } & \multicolumn{6}{|c|}{ Relative Frequency (\%) } & \multicolumn{8}{|c|}{ Average Coverage (\%) } \\
\hline & & & & AdQp & AuQp & BhQp & LbQp & StQp & DlAg & GuCa & AdQp & AuQp & BhQp & LbQp & StQp & DlAg & GuCa \\
\hline $\begin{array}{l}\text { Quercus pyrenaica Willd. } \\
\text { Acer opalus subsp. }\end{array}$ & 12.59 & Q-F & E1 & 100.00 & 97.20 & 100.00 & 100.00 & 100.00 & 1.79 & - & 53.38 & 53.20 & 45.83 & 65.00 & 72.91 & 18.50 & - \\
\hline $\begin{array}{l}\text { granatense (Boiss.) Font } \\
\text { Quer and Rothm. }\end{array}$ & 6.29 & Q-F & E2 & 26.23 & 0.70 & 38.89 & - & - & 83.04 & 29.41 & 22.38 & 45.00 & 10.29 & - & - & 35.27 & 14.10 \\
\hline Corylus avellana $\mathrm{L}$. & 5.00 & Q-F & E2 & - & - & - & - & 4.65 & 5.36 & 100.00 & - & - & - & - & 6.00 & 19.67 & 46.65 \\
\hline $\begin{array}{l}\text { Quercus faginea subsp. } \\
\text { faginea Lam. }\end{array}$ & 4.85 & Q-F & E1 & 3.28 & 13.29 & 5.56 & - & 4.65 & 48.21 & 47.06 & 12.00 & 21.00 & 25.00 & - & 6.00 & 28.19 & 16.25 \\
\hline $\begin{array}{l}\text { Brachypodium sylvaticum } \\
\text { (Huds.) P. Beauv. }\end{array}$ & 4.37 & S-P & E3 & 4.92 & 27.27 & 16.67 & - & 27.91 & 32.14 & 44.12 & 8.00 & 12.87 & 20.67 & - & 10.08 & 19.00 & 23.87 \\
\hline $\begin{array}{l}\text { Rubia peregrina subsp. } \\
\text { peregrina } \mathrm{L} .\end{array}$ & 4.32 & Q-I & E2 & 13.11 & 37.06 & 61.11 & - & 9.30 & 30.36 & 41.18 & 11.38 & 10.21 & 16.91 & - & 6.00 & 12.21 & 10.79 \\
\hline $\begin{array}{l}\text { Daphne laureola subsp. } \\
\text { latifolia (Coss.) Rivas Mart. }\end{array}$ & 4.30 & Q-F & E2 & 14.75 & - & 44.44 & - & - & 53.57 & 41.18 & 37.67 & - & 14.63 & - & - & 17.57 & 16.71 \\
\hline Arbutus unedo L. & 4.26 & Q-I & E2 & - & 50.35 & - & 90.00 & 16.28 & 2.68 & - & - & 20.39 & - & 9.33 & 13.14 & 8.00 & - \\
\hline Helleborus foetidus $\mathrm{L}$. & 4.10 & Q-F & E3 & 36.07 & 0.70 & 44.44 & - & - & 50.89 & 79.41 & 8.73 & 25.00 & 12.13 & - & - & 13.46 & 10.22 \\
\hline Daphne gnidium L. & 3.64 & Q-I & E2 & 11.48 & 46.15 & 5.56 & 40.00 & 23.26 & 7.14 & 14.71 & 9.43 & 10.62 & 12.00 & 7.50 & 7.80 & 8.25 & 7.20 \\
\hline $\begin{array}{l}\text { Quercus faginea subsp. } \\
\text { alpestris (Boiss.) Maire }\end{array}$ & 3.55 & Q-F & E1 & - & 0.70 & 83.33 & - & - & 5.36 & 2.94 & - & 45.00 & 26.00 & - & - & 43.00 & 25.00 \\
\hline Tamus communis $\mathrm{L}$. & 3.47 & Q-F & E2 & - & 37.76 & - & - & 32.56 & 15.18 & 23.53 & - & 11.04 & - & - & 10.36 & 11.00 & 9.00 \\
\hline $\begin{array}{c}\text { Paeonia broteri Boiss. \& } \\
\text { Reut. }\end{array}$ & 3.45 & Q-I & E3 & - & 32.87 & 11.11 & - & 53.49 & 16.96 & - & - & 11.99 & 9.00 & - & 13.87 & 7.42 & - \\
\hline Hedera helix subsp. helix $\mathrm{L}$. & 3.36 & Q-F & E2 & 8.20 & 2.10 & 50.00 & - & 6.98 & 29.46 & 67.65 & 6.00 & 16.33 & 12.89 & - & 8.00 & 11.42 & 16.35 \\
\hline Quercus suber var. suber L. & 3.34 & Q-I & E1 & - & 35.66 & - & 60.00 & - & - & - & - & 18.95 & - & 10.00 & - & - & - \\
\hline Myrtus communis L. & 3.04 & $\hat{\mathrm{Q}}-\mathrm{I}$ & E2 & - & 2.10 & - & - & - & - & - & - & 31.67 & - & - & - & - & - \\
\hline $\begin{array}{l}\text { Aristolochia paucinervis } \\
\text { Pomel }\end{array}$ & 2.97 & S-P & E3 & 26.23 & 20.28 & 11.11 & - & 41.86 & 8.04 & - & 9.75 & 9.52 & 6.00 & - & 14.28 & 11.44 & - \\
\hline $\begin{array}{l}\text { Quercus broteroi (Cout.) } \\
\text { Rivas Mart. }\end{array}$ & 2.88 & Q-I & E1 & - & 20.28 & - & - & 13.95 & - & - & - & 22.02 & - & - & 13.17 & - & - \\
\hline $\begin{array}{l}\text { Acer monspessulanum } \\
\text { subsp. monspessulanum } \mathrm{L} \text {. }\end{array}$ & 2.88 & Q-F & E2 & - & 15.38 & - & - & 20.93 & 20.54 & - & - & 14.10 & - & - & 15.11 & 15.78 & - \\
\hline Primula acaulis (L.) L. & 2.80 & Q-F & E3 & - & 0.70 & 44.44 & - & 6.98 & 25.89 & 73.53 & - & 6.00 & 13.63 & - & 6.00 & 10.38 & 13.48 \\
\hline Erica arborea L. & 2.79 & Q-I & E2 & - & 27.27 & - & 30.00 & 27.91 & - & - & - & 14.67 & - & 10.00 & 9.00 & - & - \\
\hline $\begin{array}{l}\text { Teucrium scorodonia subsp. } \\
\text { scorodonia L. }\end{array}$ & 2.79 & Q-F & E3 & - & 33.57 & - & - & 44.19 & - & - & - & 11.04 & - & - & 10.58 & - & - \\
\hline Ilex aquifolium $\mathrm{L}$. & 2.76 & Q-F & E2 & - & 0.70 & 55.56 & 10.00 & 11.63 & 12.50 & 52.94 & - & 12.00 & 14.10 & 12.00 & 9.60 & 9.50 & 22.28 \\
\hline Doronicum plantagineum $\mathrm{L}$. & 2.74 & Q-I & E3 & 54.10 & 12.59 & - & - & 44.19 & - & - & 6.73 & 8.72 & - & - & 10.53 & - & - \\
\hline Sorbus aria (L.) Crantz & 2.70 & Q-F & E1 & 24.59 & - & 22.22 & - & 13.95 & 30.36 & 8.82 & 16.13 & - & 13.75 & - & 10.00 & 10.53 & 8.00 \\
\hline
\end{tabular}


Table A1. Cont.

\begin{tabular}{|c|c|c|c|c|c|c|c|c|c|c|c|c|c|c|c|c|c|}
\hline \multirow{2}{*}{ Taxon } & \multirow{2}{*}{ IVI } & \multirow{2}{*}{$\begin{array}{l}\text { Phytosociological } \\
\text { Class }\end{array}$} & \multirow{2}{*}{ Stratum } & \multicolumn{6}{|c|}{ Relative Frequency (\%) } & \multicolumn{8}{|c|}{ Average Coverage (\%) } \\
\hline & & & & AdQp & AuQp & BhQp & LbQp & StQp & DlAg & GuCa & AdQp & AuQp & BhQp & LbQp & StQp & DlAg & GuCa \\
\hline $\begin{array}{l}\text { Quercus coccifera subsp. } \\
\text { coccifera L. } \\
\text { Luzula forsteri subsp. }\end{array}$ & 2.67 & Q-I & E2 & - & 4.20 & - & - & - & 5.36 & - & - & 28.33 & - & - & - & 19.67 & - \\
\hline $\begin{array}{c}\text { forsteri (Sm.) DC. in Lam. } \\
\text { \& DC. }\end{array}$ & 2.67 & Q-F & E3 & 21.31 & 13.29 & - & - & 72.09 & - & - & 8.85 & 9.84 & - & - & 12.16 & - & - \\
\hline Pistacia terebinthus L. & 2.41 & Q-I & E2 & 1.64 & 6.29 & - & - & 6.98 & 17.86 & 11.76 & 12.00 & 11.57 & - & - & 14.33 & 17.60 & 9.00 \\
\hline Ruscus aculeatus L. & 2.30 & Q-I & E3 & - & 25.17 & - & - & 11.63 & 5.36 & 2.94 & - & 11.48 & - & - & 6.00 & 15.33 & 12.00 \\
\hline $\begin{array}{l}\text { Holcus mollis subsp. mollis } \\
\text { L. }\end{array}$ & 2.20 & Q-F & E3 & 3.28 & 11.19 & - & 20.00 & 16.28 & - & - & 6.00 & 16.50 & - & 6.00 & 19.71 & - & - \\
\hline Carex distachya Desf. & 2.16 & Q-I & E3 & 3.28 & 13.99 & - & 50.00 & 39.53 & - & - & 6.00 & 9.35 & - & 10.80 & 12.53 & - & - \\
\hline $\begin{array}{l}\text { Piptatherum paradoxum (L.) } \\
\text { P. Beauv. }\end{array}$ & 2.13 & Q-I & E3 & - & - & 5.56 & - & - & 14.29 & 17.65 & - & - & 6.00 & - & - & 19.31 & 11.00 \\
\hline Pistacia lentiscus L. & 2.11 & Q-I & E2 & - & - & - & - & - & 3.57 & - & - & - & - & - & - & 21.75 & - \\
\hline Phillyrea angustifolia L. & 2.09 & Q-I & E2 & - & 27.97 & - & - & - & 0.89 & - & - & 10.93 & - & - & - & 6.00 & - \\
\hline $\begin{array}{l}\text { Juniperus oxycedrus subsp. } \\
\text { oxycedrus L. }\end{array}$ & 1.89 & Q-I & E2 & - & 8.39 & 5.56 & - & 9.30 & 16.07 & 2.94 & - & 11.58 & 12.00 & - & 6.00 & 8.33 & 12.00 \\
\hline $\begin{array}{c}\text { Paeonia officinalis subsp. } \\
\text { microcarpa (Boiss. \& Reut.) } \\
\text { Nyman }\end{array}$ & 1.82 & Q-F & E3 & - & - & 33.33 & - & 6.98 & 17.86 & 11.76 & - & - & 9.00 & - & 10.00 & 10.55 & 6.00 \\
\hline Rosa sicula Tratt. & 1.81 & Q-I & E2 & 1.64 & - & - & - & - & 12.50 & - & 12.00 & - & - & - & - & 14.50 & - \\
\hline Smilax aspera L. & 1.79 & Q-I & E2 & - & 6.29 & - & 30.00 & 4.65 & 2.68 & - & - & 14.33 & - & 10.00 & 9.00 & 14.33 & - \\
\hline $\begin{array}{c}\text { Rhamnus alaternus subsp. } \\
\text { alaternus } \mathrm{L} \text {. }\end{array}$ & 1.76 & Q-I & E2 & - & 2.80 & - & - & - & 5.36 & - & - & 21.75 & - & - & - & 12.17 & - \\
\hline $\begin{array}{l}\text { Olea europaea var. sylvestris } \\
\text { (Mill.) Lehr }\end{array}$ & 1.75 & Q-I & E2 & - & 4.90 & - & - & - & 0.89 & - & - & 18.57 & - & - & - & 6.00 & - \\
\hline Euphorbia characias L. & 1.67 & Q-I & E3 & 14.75 & 0.70 & - & - & - & 2.68 & - & 12.00 & 25.00 & - & - & - & 10.00 & - \\
\hline $\begin{array}{c}\text { Fraxinus angustifolia subsp. } \\
\text { angustifolia Vahl }\end{array}$ & 1.66 & S-P & E1 & 4.92 & 3.50 & - & - & - & 8.93 & 20.59 & 10.00 & 16.00 & - & - & - & 7.20 & 12.29 \\
\hline $\begin{array}{l}\text { Bunium macuca subsp. } \\
\text { macuсa Boiss. }\end{array}$ & 1.64 & Q-F & E3 & - & - & - & - & - & 3.57 & - & - & - & - & - & - & 17.00 & - \\
\hline Sanicula europaea L. & 1.63 & Q-F & E3 & - & - & 16.67 & - & 11.63 & - & 5.88 & - & - & 12.00 & - & 12.20 & - & 18.50 \\
\hline Melica uniflora Retz. & 1.59 & $\mathrm{Q}-\mathrm{F}$ & E3 & - & - & - & - & 6.98 & - & - & - & - & - & - & 16.33 & - & - \\
\hline $\begin{array}{l}\text { Viola sylvestris subsp. } \\
\text { sylvestris Lam. }\end{array}$ & 1.51 & Q-F & E3 & - & - & 16.67 & - & - & 9.82 & 8.82 & - & - & 14.33 & - & - & 10.36 & 16.33 \\
\hline Asplenium onopteris L. & 1.48 & Q-I & E3 & - & 15.38 & - & - & - & - & - & - & 8.59 & - & - & - & - & - \\
\hline Taxus baccata L. & 1.46 & Q-F & E1 & 1.64 & - & 16.67 & - & 6.98 & 8.04 & 14.71 & 6.00 & - & 8.00 & - & 10.00 & 9.11 & 8.40 \\
\hline $\begin{array}{l}\text { Vincetoxicum nigrum (L.) } \\
\text { Moench }\end{array}$ & 1.46 & Q-I & E3 & - & 13.29 & 5.56 & - & 11.63 & 7.14 & - & - & 7.63 & 6.00 & - & 7.20 & 6.00 & - \\
\hline $\begin{array}{l}\text { Conopodium pyrenaeum } \\
\text { (Loisel.) Miégev. }\end{array}$ & 1.44 & Q-F & E3 & 8.20 & - & 11.11 & - & 11.63 & 7.14 & - & 8.40 & - & 6.00 & - & 9.60 & 9.75 & - \\
\hline Paeonia coriacea Boiss. & 1.39 & Q-I & E3 & 24.59 & - & - & - & - & 2.68 & - & 9.20 & - & - & - & - & 8.00 & - \\
\hline
\end{tabular}


Table A1. Cont.

\begin{tabular}{|c|c|c|c|c|c|c|c|c|c|c|c|c|c|c|c|c|c|}
\hline \multirow{2}{*}{ Taxon } & \multirow{2}{*}{ IVI } & \multirow{2}{*}{$\begin{array}{l}\text { Phytosociological } \\
\text { Class }\end{array}$} & \multirow{2}{*}{ Stratum } & \multicolumn{6}{|c|}{ Relative Frequency (\%) } & \multicolumn{8}{|c|}{ Average Coverage (\%) } \\
\hline & & & & AdQp & $\mathrm{AuQp}$ & BhQp & LbQp & StQp & DIAg & GuCa & AdQp & $\mathrm{AuQp}$ & BhQp & LbQp & StQp & DlAg & GuCa \\
\hline $\begin{array}{l}\text { Lathyrus linifolius } \\
\text { (Reichard) Bässler }\end{array}$ & 1.37 & Q-F & E3 & - & 0.70 & - & - & 27.91 & - & - & - & 6.00 & - & - & 11.75 & - & - \\
\hline Lonicera implexa Aiton & 1.36 & Q-I & E2 & - & 6.99 & - & - & - & 6.25 & - & - & 7.22 & - & - & - & 11.29 & - \\
\hline Genista tournefortii Spach & 1.32 & Q-I & E3 & - & 9.79 & - & - & 2.33 & - & - & - & 9.50 & - & - & 6.00 & - & - \\
\hline $\begin{array}{l}\text { Sanguisorba hybrida (L.) } \\
\text { Font Quer }\end{array}$ & 1.30 & Q-I & E3 & - & 5.59 & - & - & 4.65 & - & - & - & 13.00 & - & - & 6.00 & - & - \\
\hline Genista falcata Brot. & 1.28 & Q-F & E3 & - & 2.10 & - & - & 30.23 & - & - & - & 10.00 & - & - & 7.38 & - & - \\
\hline Hepatica nobilis Schreb. & 1.22 & $\mathrm{Q}-\mathrm{F}$ & E3 & - & - & - & - & 9.30 & - & 8.82 & - & - & - & - & 6.00 & - & 18.67 \\
\hline Pyrus bourgaeana Decne. & 1.17 & Q-I & E2 & - & 4.20 & - & - & - & - & - & - & 11.00 & - & - & - & - & - \\
\hline $\begin{array}{l}\text { Asparagus aphyllus subsp. } \\
\text { aphyllus L. }\end{array}$ & 1.16 & Q-I & E2 & - & 2.80 & - & - & - & - & - & - & 12.00 & - & - & - & - & - \\
\hline Potentilla sterilis (L.) Garcke & 1.14 & Q-F & E3 & - & - & - & - & 6.98 & - & - & - & - & - & - & 12.00 & - & - \\
\hline Veronica officinalis L. & 1.09 & Q-F & E3 & - & - & 11.11 & - & 6.98 & - & 8.82 & - & - & 9.00 & - & 6.00 & - & 10.00 \\
\hline $\begin{array}{l}\text { Bupleurum rigidum subsp. } \\
\text { paniculatum (Brot.) H. } \\
\text { Wolff. }\end{array}$ & 1.08 & Q-I & E3 & - & 4.20 & - & - & 2.33 & - & - & - & 8.00 & - & - & 12.00 & - & - \\
\hline $\begin{array}{l}\text { Dryopteris affinis subsp. } \\
\text { affinis (Lowe) Fraser-Jenk. }\end{array}$ & 1.07 & Q-F & E3 & - & - & - & - & 11.63 & - & - & - & - & - & - & 9.60 & - & - \\
\hline Ulmus glabra Huds. & 1.07 & Q-F & E1 & - & - & - & - & - & 2.68 & 8.82 & - & - & - & - & - & 8.00 & 10.00 \\
\hline Euphorbia hyberna L. & 1.04 & Q-F & E3 & - & 0.70 & - & - & 4.65 & - & - & - & 12.00 & - & - & 9.00 & - & - \\
\hline Carex pendula Huds. & 1.02 & S-P & E3 & - & - & - & - & - & 0.89 & 8.82 & - & - & - & - & - & 6.00 & 10.00 \\
\hline Senecio lopezii Boiss. & 1.02 & Q-I & E3 & - & - & - & 40.00 & - & - & - & - & - & - & 9.00 & - & - & - \\
\hline $\begin{array}{l}\text { Ajuga x_rotundifolia Willk. } \\
\text { \& Cutanda ex Willk. }\end{array}$ & 0.97 & Q-F & E3 & - & - & - & - & 4.65 & - & - & - & - & - & - & 9.00 & - & - \\
\hline Carex sylvatica Huds. & 0.95 & Q-F & E3 & - & - & - & - & - & - & 11.76 & - & - & - & - & - & - & 7.50 \\
\hline Moehringia pentandra J. Gay & 0.92 & Q-I & E4 & - & 6.99 & - & - & 11.63 & - & - & - & 5.70 & - & - & 6.00 & - & - \\
\hline Asparagus acutifolius L. & 0.91 & Q-I & E2 & - & 9.09 & - & - & - & 4.46 & - & - & 6.69 & - & - & - & 13.40 & - \\
\hline $\begin{array}{l}\text { Phillyrea latifolia subsp. } \\
\text { latifolia } \mathrm{L} .\end{array}$ & 0.86 & Q-I & E2 & - & 4.20 & - & - & - & 2.68 & 8.82 & - & 3.55 & - & - & - & 10.00 & 8.00 \\
\hline $\begin{array}{l}\text { Juniperus phoenicea subsp. } \\
\text { phoenicea L. }\end{array}$ & 0.78 & Q-I & E2 & - & - & - & - & - & 6.25 & 2.94 & - & - & - & - & - & 6.86 & 6.00 \\
\hline Clematis campaniflora Brot. & 0.72 & S-P & E2 & - & 3.50 & - & - & - & - & - & - & 7.20 & - & - & - & - & - \\
\hline $\begin{array}{l}\text { Epipactis microphylla (Ehrh.) } \\
\text { Sw. }\end{array}$ & 0.66 & Q-F & E3 & - & - & - & - & - & 0.89 & 11.76 & - & - & - & - & - & 6.00 & 6.00 \\
\hline Anemone palmata $\mathrm{L}$. & 0.63 & Q-I & E3 & - & 2.80 & - & - & - & - & - & - & 6.00 & - & - & - & - & - \\
\hline Viola suavis M. Bieb. & 0.63 & Q-F & E3 & - & - & - & - & - & 3.57 & - & - & - & - & - & - & 6.00 & - \\
\hline Iris foetidissima $\mathrm{L}$. & 0.61 & S-P & E3 & - & - & - & - & - & - & 8.82 & - & - & - & - & - & - & 6.00 \\
\hline
\end{tabular}

Taxa occurring in less than $5 \%$ in all associations have been discarded. The taxa are listed in order of importance (IVI). The phytosociological adscription of the taxa is also shown: Q-F (Querco roboris-Fagetea sylvaticae), Q-I (Quercetea ilicis), S-P (Salici purpureae-Populetea nigrae). Adenocarpo decorticantis-Quercetum pyrenaicae (AdQp); Arbuto unedonis-Quercetum pyrenaicae (AuQp); Berberido australis-Quercetum pyrenaicae (BhQp); Luzulo baeticae-Quercetum pyrenaicae (LbQp); Sorbo torminalis-Quercetum pyrenaicae (StQp); Daphno latifoliae-Aceretum granatensis (DlAg), and Geo urbani-Coryletum avellanae (GuCa). 


\section{References}

1. Piñar Fuentes, J.C.; Cano-Ortiz, A.; Musarella, C.; Pinto-Gomes, C.; Spampinato, G.; Cano, E. Rupicolous habitats of interest for conservation in the central-southern Iberian peninsula. Plant. Sociol. 2017, 54, $29-42$.

2. Molero, J.; Marfil, J.M. The bioclimates of Sierra Nevada National Park. Int. J. Geobot. Res. 2015, 5, 1-11.

3. Rivas-Martínez, S. Bioclimatoloy of the Iberian Peninsula. Itinera Geobot. 1999, 13, 41-47.

4. Cano, E.; Cano-Ortiz, A.; Musarella, C.M.; Piñar Fuentes, J.C.; Ighbareyeh, J.M.H.; Gea, F.L.; del Río, S. Mitigating Climate Change Through Bioclimatic Applications and Cultivation Techniques in Agriculture (Andalusia, Spain). In Sustainable Agriculture, Forest and Environmental Management; Springer: Singapore, 2019; pp. 31-69.

5. Rivas-Martínez, S.; Rivas-Saenz, S.; Penas, Á. Worldwide bioclimatic classification system. Glob. Geobot. 2011, 1, 1-634.

6. Valladares, F.; Pearcy, R.W. Drought can be more critical in the shade than in the sun: A field study of carbon gain and photo-inhibition in a Californian shrub during a dry El Niño year. Plant Cell Environ. 2002, 25, 749-759. [CrossRef]

7. Stanhill, G.; Cohen, S. Global dimming: A review of the evidence for a widespread and significant reduction in global radiation with discussion of its probable causes and possible agricultural consequences. Agr. For. Meteorol. 2001, 107, 255-278. [CrossRef]

8. Valladares, F. Global change and radiation in Mediterranean forest ecosystems: A meeting point for ecology and management. In Ecology, Conservation and Sustainable Management of Mediterranean Type Ecosystems of the World; Millpress: Rotterdam, The Netherlands, 2004; pp. 1-4.

9. Gómez-Aparicio, L.; Gómez, J.M.; Zamora, R. Microhabitats shift rank in suitability for seedling establishment depending on habitat type and climate. J. Ecol. 2005, 93, 1194-1202. [CrossRef]

10. Kitzberger, T.; Steinaker, D.; Veblen, T. Effects of climatic variability on facilitation of tree establishment in northern Patagonia. Ecology 2000, 81, 1914-1921. [CrossRef]

11. Schenk, H.J.; Mahal, B.E. Positive and negative plant interactions contribute to north-south-patterned association between two desert shrub species. Oecologia 2002, 132, 402-410. [CrossRef]

12. Evans, R.; Young, J. Plant litter and establishment of alien annual weed species in rangeland communities. Weed Sci. 1970, 18, 697-703. [CrossRef]

13. Fowler, N. What is a safe site? Neighbour, litter, germination date, and patch effects. Ecology 1988, 69, 947-961. [CrossRef]

14. Verdú, M.; García-Fayos, P. Nucleation processes in a Mediterranean bird-dispersed plant. Funct. Ecol. 1996, 10, 275-280. [CrossRef]

15. Callaway, R.N. Facilitation and interference of Quercus douglasii on understorey productivity in central California. Ecology 1991, 72, 1484-1499. [CrossRef]

16. Joffre, R. How tree cover influences the water balance of Mediterranean rangelands. Ecology 1993, 74, 570-882. [CrossRef]

17. Quinto-Canas, R.; Mendes, P.; Cano-Ortiz, A.; Musarella, C.M.; Pinto-Gomes, C. Comunidades de orla forestal en el suroeste de la península ibérica. Rev. Chapingo Ser. Cienc. For. Y Ambiente 2018, XXIV, 415-434.

18. Gómez, J.M.; Gómez, L.; Zamora, R.; Montes, J. Problemas de regeneración de especies forestales autóctonas en el espacio natural protegido de Sierra Nevada. In Congreso Forestal Español; Sociedad Española de Ciencias Forestales: Palenciana, Spain, 2001.

19. Aparicio, L.G. Papel de la heterogeneidad en la regeneración del Acer opalus subsp. granatense en la montaña mediterránea: Impicaciones para la conservación y restauración de sus poblaciones. Ecosistemas 2004, 13, 123-128.

20. Cano, E.; Musarella, C.M.; Cano-Ortiz, A.; Piñar Fuentes, J.C.; Rodríguez Torres, A.; Del Río González, S.; Pinto Gomes, C.P.; Quinto-Canas, R.; Spampinato, G. Geobotanical Study of the Microforests of Juniperus oxycedrus subsp. badia in the Central and Southern Iberian Peninsula. Sustainability 2019, 11, 1111. [CrossRef]

21. Valle, F.; Mota, J.F.; Gomez Mercado, F. Los Avellanares del Macizo Segura-Cazorla: Relaciones Ombroclima-Vegetación. En A Pulido, El Agua en Andalucía; Departamento de Hidrogeología: Granada, Spain, 1986; Volume 2, pp. 567-580. 
22. Vila-Viçosa, C.; Vázquez, F.M.; Mendes, P.; Del Rio, S.; Musarella, C.; Cano-Ortiz, A.; Meireles, C. Syntaxonomic update on the relict groves of Mirbeck's oak (Quercus canariensis Willd. and Q. marianica C. Vicioso) in southern Iberia. Plant Biosyst. 2015, 149, 512-526. [CrossRef]

23. Reyes, O.; Casal, M. Seed germination of Quercus robur, Q. pyrenaica and Q. ilex and the effects of smoke, heat, ash and charcoal. Ann. For. Sci. 2006, 63, 205-212. [CrossRef]

24. Itziar, R.U.; Pérez-Ramos, I.M.; Zavala, M.A.; Marañón, T.; Kobe, R.K. Soil water content and emergence time control seedling establishment in three co-occurring Mediterranean oak species. Can. J. For. Res. 2008, 38, 2382-2393.

25. Bioclimatic Map of Europe. Available online: http://www.globalbioclimatics.org/form/bi_med.htm (accessed on 15 July 2019).

26. Rivas-Martínez, S.; Penas, A.; Díaz González, T.E.; Cantó, P.; del Río, S.; Costa, J.C.; Herrero, L.; Molero Mesa, J. Biogeographic units of the iberian peninsula and baelaric islands to district level. A concise synopsis. In The Vegetation of the Iberian Peninsula Plant and Vegetation; Loidi, J., Ed.; Springer: Cham, Switzerland, 2017; Volume 12.

27. Braun-Blanquet, J. Fitosociología: Bases Para el Estudio de Las Comunidades Vegetales; Ediciones Blume: Madrid, Spain, 1979.

28. Martínez Parras, J.M.; Molero Mesa, J. Ecología y fitosociología de Quercus pyrenaica Willd. en la provincia Bética. Los melojares béticos y sus etapas de sustitución. Lazaroa 1982, 4, 91-104.

29. Rivas Martinez, S. Memoria del Mapa de Las Series de Vegetación de España: 30 pp; ICONA Ministerio de Agricultura y Medio Ambiente: Madrid, Spain, 1987.

30. Valle, F.; Mercado, F.G.; Poveda, J.F.M. Los robledales de la Sierra de Segura y otras comunidades relacionadas con ellos. Anal. Jardin. Bot. Madr. 1988, 45, 247-257.

31. Rivas Martínez, S.; González, T.E.D.; González, F.F.; Arregui, J.J.L.; Lousã, M.F.; Merino, Á.P. Vascular plant communities of Spain and Portugal: Addenda to the syntaxonomical checklis of 2001. Part 1. Itinera Geobot. 2002, 15, 5-432.

32. Rivas-Martínez, S. Esquema de la vegetación potencial y su correspondencia con los suelos en la España peninsular. Anal. Inst. Bot. Cavanilles 1964, 22, 341-405.

33. Al Aallali, A.; Nieto, J.M.L.; Raya, F.A.P.; Mesa, J.M. Estudio de la vegetación forestal en la vertiente sur de Sierra Nevada (Alpujarra Alta granadina). Itinera Geobot. 1998, 11, 387-402.

34. Losa, J.M.; Molero, J.; Casares, M.; Pérez-Raya, F. El paisaje vegetal de Sierra Nevada. La cuenca alta del río Genil. Granada. Serv. Publ. Univ. Granada 1986, 1, 1-285.

35. Nieto, J.M.; Cabezudo, B. Series de vegetación climatófilas de las sierras Tejeda y Almijara (Málaga-Granada, España). Acta Bot. Malacit. 1988, 13, 229-260.

36. Pérez Latorre, A.V.; Navas Fernández, D.; Gavira, O.; Caballero, G.; Cabezudo, B. Vegetación del Parque Natural de Las Sierras Tejeda, Almijara y Alhama (Málaga-Granada, España). Acta Bot. Malacit. 2004, 29, 117-190.

37. Junta de Andalucía. Cartografía Y Evaluación de la Vegetación Y Flora de Los Ecosistemas Forestales de Andalucía a Escala de Detalle (1:10.000); Red de Información Ambiental de Andalucía (REDIAM): Andalucia, Spain, 1996-2006.

38. Rivas Goday, S.; Borja Carbonell, J.; Esteve Chueca, F.; Fernández-Galiano, E.; Rigual Magallon, A.; Rivas Martínez, S. Contribución al estudio de la Quercetea ilicis hispánica. Anal. Inst. Bot. Cavanilles 1959, 17, $285-403$.

39. Cano Carmona, E.; Valle, F. Formaciones boscosas en Sierra Morena oriental. Acta Bot. Malacit. 1990, 15, 231-237.

40. Melendo Luque, M. Cartografía Y Ordenación vegetal de Sierra Morena: Parque Natural de Las Sierras de Cardeña Y Montoro (Córdoba). Ph.D. Thesis, Universidad Jaén, Jaen, Spain, 1998.

41. Vicente Orellana, J.A.; Galán De Mera, A. Nuevas aportaciones al Conocimiento de la vegetación Luso-Extremadurense. Estudio de las Sierras de Las Villuercas (Extremadura, España) y San Mamede (Alto Alentejo, Portugal). Acta Bot. Malacit. 2008, 33, 1-49.

42. Amor, A. Flora Y Vegetación Vascular de la Comarca de la Vera Y Laderas Meridionales de la SIERRA de Tormantos (Cáceres). Ph.D. Thesis, Universidad de Salamanca, Salamanca, Spain, 1991.

43. Belmonte, M.D. La Vegetación del Monfragüe y Su Área Socioeconómica de Influencia; Junta de Extremadura: Caceres, Spain, 2008. 
44. Monteiro-Henriques, T. Landscape and Phytosociology of the Paiva River's Hidrographical Basin. Ph.D. Thesis, Universidade Técnica de Lisboa, Lisboa, Portuguesa, 2010.

45. Pereira de Menezes Dias, M.C. A Flora e Vegetação da Serra de Monfurado (Alto Alentejo-Portugal). Guineana 2009, 15, 316.

46. Sánchez-Pascual, N. Estudio fitosociológico y cartográfico de la comarca de Despeñaperros (Jaén). Ph.D. Thesis, Universidad de Granada, Granada, Spain, 1994.

47. López Vélez, G. Flora y vegetación del macizo del Calar del Mundo y sierras adyacentes del sur de Albacete; Instituto de Estudios Albacetenses: Albacete, Spain, 1996; p. 520.

48. Herranz, J.M.; Gómez Del Pozo, E.C. Contribución al conocimiento de la flora y vegetación de la comarca de Alcaraz; Caja de Ahorros de Albacete: Albacete, Spain, 1986; p. 279.

49. Galán de Mera, A. Flora y Vegetación de los términos municipales de Alcalá de los Gazules y Medina Sidonia (Cádiz, España). Ph.D. Thesis, Universidad Complutense de Madrid, Madrid, Spain, 1993.

50. Pérez Latorre, A.V.; Galán de Mera, A.; Deil, U.; Cabezudo, B. Fitogeografía y vegetación del sector aljíbico (Cádiz-Málaga, España). Acta Bot. Malacit. 1996, 21, 241-267.

51. Asensi, A.; Díaz Garreta, B. The vegetation of Los Alcornocales Natural Park: Ronda-Faro (16 July). Itinera Geobot. 1999, 13, 129-135.

52. Rivas Goday, S. Vegetación y flórula de la cuenca extremeña del Guadiana; Publicaciones de la Excma: Madrid, Spain, 1964; p. 777.

53. Cantó, P. Estudio fitosociológico y biogeográfico de la sierra de San Vicente y tramo inferior del valle del Alberche (Toledo, España). Lazaroa 2004, 25, 187-250.

54. Cabezudo, B.; Pérez-Latorre, A.; Navas, P.; Gil, Y.; Navas, D. Parque natural de la Sierra de las Nieves. Cartografía y evaluación de la flora y vegetación. Memoria; Departamento de Biología Vegetal, Universidad de Málaga: Málaga, Spain, 1998; p. 367.

55. Giménez Luque, E. Bases Botánico-Ecológicas Para la Restauración de la Cubierta Vegetal de la Sierra de Gádor (Almería). Ph.D. Thesis, Universidad de Almería, Almería, Spain, 2004.

56. Gómez Mercado, F.; Valle Tendero, F. Mapa de Vegetación de La Sierra de Baza; Servicio de Publicaciones: Madrid, Spain, 1988.

57. Mercado, F.G. Vegetación y flora de la Sierra de Cazorla. Guineana 2011, 17, 1-481.

58. Gómez Mercado, F.; Mota, J.F.; Peñas, J.; Cabello, J.; Valle, F. Vegetación. In Reconocimiento Biofísico de Espacios Naturales Protegidos. Parque Natural Sierras Subbéticas; Moreira, Ed.; Consejería de Medio Ambiente, Junta de Andalucía: Sevilla, Spain, 2000; pp. 195-311.

59. Madrona, M.T. Cartografía de la vegetación actual y planificación de la restauración vegetal en las sierras de Lújar y la Contraviesa. Ph.D. Thesis, Universidad de Granada, Granada, Spain, 1994.

60. Pavón Nuñez, M.; Pérez Latorre, A.V. Sobre las avellanedas subbéticas con Ulmus glabra Huds. en las sierras de Cazorla-Segura-Alcaráz, Jaen-Albacete (España). Lagascalia 2010, 30, $393-405$.

61. Pérez-Latorre, A.V.; Casimiro-Soriguer, F.; Gavira, O.; Cabezudo, B. Vegetación de la reserva de la biosfera Sierra de las Nieves: Río Grande y Sierras Prieta y Blanquilla (Málaga, España). Acta Bot. Malacit. 2012, 37, 103-140. [CrossRef]

62. Inocencio, C.; Alcaraz, F.J.; Ríos, S. El paisaje vegetal de la Cuenca albacetense del Guadalmena. Inst. Estud. Albacet. Excma. Diput. Albacete Ser. I 1998, 100, 327.

63. Olmedo, J.A. Análisis Biogeográfico y Cartografía de la Vegetación de la Sierra de Baza (Provincia de Granada). Ph.D. Thesis, Universidad de Granada, Granada, Spain, 2011.

64. Gómez Navarro, J. A portaciones al estudio de la flora y vegetación del extremo NE de la provincia de Albacete y zonas adyacentes de la provincia de Valencia (España). Fac. Ciènc. Biol. Univ. València. 2008, 2, 457-927.

65. Molina Cantos, R.; Valdés Franzi, A.; Alcaraz Ariza, F.J. Flora y Vegetación del Tramo Medio del Valle del Río Júcar (Albacete); Instituto de Estudios Albacetenses ‘Don Juan Manuel' de la Excma; Diputación de Albacete: Albacete, Spain, 2008; p. 663.

66. Pavón Núñez, M.; Pérez Latorre, A.V.; Hidalgo Triana, N. Novedades fitosociológicas y florísticas en el Parque Natural de Tejeda-Almijara y Alhama (Málaga-Granada, España). Acta Bot. Malacit. 2015, 40, 199-205. [CrossRef]

67. Ríos Ruiz, S.; Alcaraz Ariza, F.; Valdés Franzi, A. Vegetación de Sotos Y Riberas de la Provincia de Albacete; Instituto de Estudios Albacetenses 'Don Juan Manuel': Albacetenses, Spain, 2003; p. 365. 
68. Vlieger, J. Aperçu sur les unités phytosociologiques supérieures des Pays-Bas. Ned. Kruidkd. Archief Ser. 3 1937, 47, 335-353.

69. Bolòs, O.; de Bolòs Vayreda, A. Vegetación de Las Comarcas Barcelonesas: Descripción Geobotánica Y Catálogo Florístico Según Estudios Efectuados Por el Propio Autor y Por Oriol de Bolós Y Capdevilla; Instituto Español de Estudios Mediterráneos: Barcelona, Spain, 1950; p. 619.

70. Rivas-Martínez, S.; Fernández-González, F.; Loidi, J.; Lousã, M.; Penas, A. Syntaxonomical checklist of vascular plant communities of Spain and Portugal to association level. Itinera Geobot. 2001, 14, 341.

71. Rivas-Martínez, S. Vascular plant communities of Spain and Portugal (addenda to the syntaxonomical checklist of 2001, part II). Itinera Geobot. 2002, 15, 433-922.

72. Del Pozo, A.; Brunel-Saldias, N.; Engler, A.; Ortega-Farias, S.; Acevedo-Opazo, C.; Lobos, G.A.; Jara-Rojas, R.; Molina-Montenegro, M.A. Climate Change Impacts and Adaptation Strategies of Agriculture in Mediterranean-Climate Regions (MCRs). Sustainability 2019, 11, 2769. [CrossRef]

73. Del Río González, S. El cambio climático y su influencia en la vegetación de Castilla y León (España). Itinera Geobot. 2005, 16, 5-534.

74. Del Río, S.; Penas, A.; Perez-Romero, R. Potential areas of deciduous forests in Spain (Castile and Leon) according to future climate change. Plant Biosyst. 2005, 139, 222-233. [CrossRef]

75. Del Río, S.; Álvarez-Esteban, R.; Cano, E.; Pinto-Gomes, C.; Penas, Á. Potential impacts of climate change on habitat suitability of Fagus sylvatica L. forests in spain. Plant Biosyst. 2018, 152, 1205-1213. [CrossRef]

76. Cano Ortiz, A.; Piñar Fuentes, J.C.; Pinto Gomes, C.J.; Musarella, C.M.; Cano, E. Expansion of the Juniperus Genus due to Anthropic Activity. In Old-Growth Forests and Coniferous Forests: Ecology, Habitat and Conservation; Serie Environmental Research Advances; Weber, R.P., Ed.; Nova Science Publishers, Inc.: Hauppauge, NY, USA, 2015; pp. 55-65.

77. Torres, J.A.; Valle, F.; Pinto, C.; García-Fuentes, A.; Salazar, C.; Cano, E. Arbutus unedo L. communities in southern Iberian Peninsula mountains. Plant Ecol. 2002, 160, 207-223. [CrossRef]

78. Torres, J.A.; Cano Carmona, E.; García Fuentes, A. Aportaciones al estudio fitosociológico de las comunidades de boj (Buxus sempervirens) en el sector subbético (Andalucía, España). Acta Bot. Malacit. 1996, 21, 319-321.

79. Rivas-Martínez, S. La vegetación de la clase Quercetea ilicis en España y Portugal. Anal. Inst. Bot. Cavanilles 1974, 31, 205-259.

80. Cano, E.; Rodríguez-Torres, A.; Pinto Gomes, C.J.; García-Fuentes, A.; Torres, J.A.; Salazar, C.; Ruiz-Valenzuela, L.; Cano-Ortiz, A.; Montilla, R.J. Analysis of the Juniperus oxycedrus L. communities in the centre and south of the Iberian peninsula (Spain and Portugal). Acta Bot. Gallica 2007, 154, 79-99. [CrossRef]

81. Pavón Núñez, M.; Hidalgo Triana, N.; Pérez Latorre, A.V. Aportaciones al conocimiento de las comunidades de Pistacia terenbinthus L. y de Acer monspessulanum L. en el Sur de la Península Ibérica. Lagascalia 2013, 33, 299-311.

82. Linares, J.C.; Carreira, J.A. El pinsapo, abeto endémico andaluz. O, ¿ Qué hace un tipo como tú en un sitio como éste? Rev. Ecosistemas 2006, 15, 171-191.

83. Carrión, J.S.; Errikarta, I.Y.; Walker, M.J.; Legaz, A.J.; Chaín§, C.; López, A. Glacial refugia of temperate, Mediterranean and Ibero-North African flora in south-eastern Spain: New evidence from cave pollen at two Neanderthal man sites. Glob. Ecol. Biogeogr. 2003, 12, 119. [CrossRef]

84. Willis, K.; Niklas, K. The role of Quaternary environmental change in plant macroevolution: The exception or the rule? Philos. Trans. R. Soc. Lond. 2004, 359, 159-172. [CrossRef]

85. Costa, J.C.; Neto, C.; Aguiar, C.; Capelo, J.; Espírito Santo, M.D.; Honrado, J.; Lousã, M. Vascular plant communities in Portugal (Continental, the Azores and Madeira). Glob. Geobot. 2012, 2, 1-180.

86. Shorohova, E.; Kuuluvainen, T.; Kangur, A.; Jõgiste, K. Natural stand structures, disturbance regimes and successional dynamics in the Eurasian boreal forests: A review with special reference to Russian studies. Ann. For. Sci. 2009, 66, 1-20. [CrossRef]

87. Strandberg, B.; Kristiansen, S.M.; Tybirk, K. Dynamic oak-scrub to forest succession: Effects of management on understorey vegetation, humus forms and soils. For. Ecol. Manag. 2005, 211, 318-328. [CrossRef]

88. Cano-Ortiz, A.; Pinto-Gomes, C.; Quinto-Canas, R.; Vila-Viçosa, C.; Rodríguez-Torres, A.; Redondo, M.M. Current state of the Prunetalia spinosae communities in the centre and south of the Iberian Peninsula (Spain, Portugal). Acta Bot. Gallica 2012, 159, 211-221. [CrossRef]

89. Castro, J.; Zamora, R.; Hódar, J.A. Restoring Quercus pyrenaica forests using pioneer shrubs as nurse plants. Appl. Veg. Sci. 2006, 9, 137-142. [CrossRef] 
90. Ajbilou, R.; Marañón, T.; Arroyo, J. Ecological and biogeographical analyses of Mediterranean forests of northern Morocco. Acta Oecol. 2006, 29, 104-113. [CrossRef]

91. Aguirre, P.V. Estudio Integral de Los Bosques de Quercus Pyrenaica Willd. en la Península Ibérica: Características Bioclimáticas, Sintaxonómicas Y Especies Indicadoras Comprehensive Study of Quercus Pyrenaica Willd. Forests at Iberian Peninsula: Indicator Species, Bioclimatic, and Syntaxonomical. Ph.D. Thesis, Universidad Complutense de Madrid, Madrid, Spain, 2014.

92. Aguilar Alba, M. Cambios y tendencias recientes en las precipitaciones de Andalucía. Cambio Clim. Andal. Evol. Y Consecuencias Medioambient. 2007, 99-116.

93. Del Río, S.; Herrero, L.; Fraile, R.; Penas, A. Spatial distribution of recent rainfall trends in Spain (1961-2006). Int. J. Climatol. 2011, 31, 656-667.

94. Del Río, S.; Cano-Ortiz, A.; Herrero, L.; Penas, A. Recent trends in mean maximum and minimum air temperatures over Spain (1961-2006). Theor. Appl. Clim. 2012, 109, 605-626. [CrossRef]

95. Alexander, L.V.; Zhang, X.; Peterson, T.C.; Caesar, J.; Gleason, B.; Klein Tank, A.M.G.; Tagipour, A. Global observed changes in daily climate extremes of temperature and precipitation. J. Geophys. Res. Atmos. 2006, 111. [CrossRef]

96. Shestakova, T.A.; Gutiérrez, E.; Kirdyanov, A.V.; Camarero, J.J.; Génova, M.; Knorre, A.A.; Voltas, J. Forests synchronize their growth in contrasting Eurasian regions in response to climate warming. Proc. Natl. Acad. Sci. USA 2016, 113, 662-667. [CrossRef]

97. Linares, J.C.; Pazo Sarria, R.; Taïqui, L.; Camarero, J.J.; Ochoa, V.; Lechuga, V.; Seco, J.I.; Viñegla, B.; Sangüesa-Barreda, G.; Gilarte, P.; et al. Efectos de las tendencias climáticas y la degradación del hábitat sobre el decaimiento de los cedrales (Cedrus atlantica) del norte de Marruecos. Ecosistemas 2012, 21, 7-14. [CrossRef]

98. IPCC. Climate Change 2014: Synthesis Report. Contribution of Working Groups I, II and III to the Fifth Assessment Report of the Intergovernmental Panel on Climate Change; Pachauri, R.K., Meyer, L.A., Eds.; IPCC: Geneva, Switzerland, 2014; p. 151.

99. Loidi, J.; Biurrun, I.; Campos, J.A.; García-Baquero, G.; García-Mijangos, I.; Herrera, M. Climate change and its influence on plant species and terrestrial habitats. Int. J. Geobot. Res. 2012, 2, 13-19. [CrossRef]

100. El Consejo, D.L.C.E. Directiva 92/43/CEE del Consejo, de 21 de mayo de 1992, relativa a la conservación de los hábitats naturales y de la fauna y flora silvestres. D. Of. Comunidades Eur. 1992, 7-50.

101. Campos, J.A.; Liendo, D.; Horvat, V.; Villasante, J.; Biurrun, I.; García-Mijangos, I.; Herrera, M. Efectividad de la red Natura 2000 en la conservación de la flora y los hábitats amenazados. In Proceedings of the Conference: I Congreso de la Sociedad Española de Geobotánica. Conservación de Hábitats Naturales en Europa, Gijon, Spain, 8-11 July 2014.

102. Nunes, L.J.; Meireles, C.I.; Pinto Gomes, C.J.; Almeida Ribeiro, N. The Evolution of Climate Changes in Portugal: Determination of Trend Series and Its Impact on Forest Development. Climate 2019, 7, 78. [CrossRef]

103. Zhang, L.; Dawes, W.R.; Walker, G.R. Response of mean annual evapotranspiration to vegetation changes at catchment scale. Water Resour. Res. 2001, 37, 701-708. [CrossRef]

104. Goulden, M.L.; Bales, R.C. Mountain runoff vulnerability to increased evapotranspiration with vegetation expansion. Proc. Natl. Acad. Sci. USA 2014, 111, 14071-14075. [CrossRef] [PubMed]

105. Cooper, D.J.; Sanderson, J.S.; Stannard, D.I.; Groeneveld, D.P. Effects of long-term water table drawdown on evapotranspiration and vegetation in an arid region phreatophyte community. J. Hydrol. 2006, 325, 21-34. [CrossRef]

106. Alcántara, J.M.; Rey, P.J.; Manzaneda, A.J. A model of plant community dynamics based on replacement networks. J. Veg. Sci. 2015, 26, 524-537. [CrossRef]

(C) 2019 by the authors. Licensee MDPI, Basel, Switzerland. This article is an open access article distributed under the terms and conditions of the Creative Commons Attribution (CC BY) license (http://creativecommons.org/licenses/by/4.0/). 